TRANSACTIONS OF THE

AMERICAN MATHEMATICAL SOCIETY

Volume 355, Number 6, Pages $2263-2303$

S 0002-9947(03)03196-9

Article electronically published on January 28, 2003

\title{
NON-CROSSING CUMULANTS OF TYPE B
}

\author{
PHILIPPE BIANE, FREDERICK GOODMAN, AND ALEXANDRU NICA
}

\begin{abstract}
We establish connections between the lattices of non-crossing partitions of type B introduced by V. Reiner, and the framework of the free probability theory of D. Voiculescu.

Lattices of non-crossing partitions (of type A, up to now) have played an important role in the combinatorics of free probability, primarily via the noncrossing cumulants of R. Speicher. Here we introduce the concept of noncrossing cumulant of type B; the inspiration for its definition is found by looking at an operation of "restricted convolution of multiplicative functions", studied in parallel for functions on symmetric groups (in type A) and on hyperoctahedral groups (in type B).

The non-crossing cumulants of type B live in an appropriate framework of "non-commutative probability space of type B", and are closely related to a type B analogue for the R-transform of Voiculescu (which is the free probabilistic counterpart of the Fourier transform). By starting from a condition of "vanishing of mixed cumulants of type B", we obtain an analogue of type B for the concept of free independence for random variables in a non-commutative probability space.
\end{abstract}

\section{INTRODUCTION}

The free probability theory of D. Voiculescu is rooted in operator algebras, but has connections to several other fields of mathematics, and in particular has a substantial combinatorial side. The combinatorics of free probability is intimately related to the Möbius inversion theory in the lattices of non-crossing partitions first studied by G. Kreweras [11. The role of non-crossing partitions in free probability was discovered by R. Speicher [17, and was the subject of fairly intensive research after that. It is noteworthy that the very concept of free independence for a family of non-commutative random variables can be formulated in terms of the concept of "non-crossing cumulants" introduced in [17].

In another direction of development in the study of non-crossing partitions, V. Reiner [16] has introduced (with motivation from problems on arrangements of hyperplanes) a type B analogue $N C^{(B)}(n)$ for the lattice $N C^{(A)}(n)$ of non-crossing partitions of $\{1, \ldots, n\}$.

The content of the present paper can be summarized in one sentence as follows: Starting from the lattices $N C^{(B)}(n)$ of Reiner, we work out the framework of what should be a "non-commutative probability space of type B", and, based on the type $\mathrm{B}$ analogue for non-crossing cumulants, we propose a concept of free independence of type B.

Received by the editors May 15, 2002 and, in revised form, September 18, 2002.

2000 Mathematics Subject Classification. Primary 46L54, 05E15, 20F55. 
Let us now elaborate. As mentioned before, the concept of free independence (of type A) can be described in terms of the non-crossing cumulants of Speicher, via a condition called "the vanishing of mixed cumulants" (see review in Section 4.3 below). So if one wants to take a combinatorial approach to free independence of type B, then the natural line of attack goes by introducing the type B analogue of the non-crossing cumulants, and by formulating the corresponding condition of vanishing of mixed cumulants. But this line of attack presents us with an immediate difficulty, that we do not have the framework where the non-crossing cumulants of type B are to be defined. This framework (the "non-commutative probability space of type B") has to be invented at the same time with the cumulants.

However, when looking at the theory in type A, one sees that the non-crossing cumulants are closely related to Voiculescu's concept of R-transform (which is the free probabilistic counterpart of the Fourier transform), and to a certain operation of "boxed convolution", 太, on power series. So one can start the attack by defining the type B analogue ${ }^{(B)}$ for $\circledast$. This turns out to be feasible (in a canonical way, in fact) for the following reason: there exists a common idea, of studying a concept of "convolution of multiplicative functions", which produces both 囚 ( = $\left.\otimes^{(A)}\right)$ and $\mathrm{X}^{(B)}$ by appropriate particularizations. In the present paper we choose to present the convolution of multiplicative functions in the framework of Cayley graphs of groups: the particularization that leads to $\star^{(A)}$ is obtained by considering symmetric groups, and the one that leads to $\otimes^{(B)}$ is obtained by considering hyperoctahedral groups. The approach in terms of Cayley graphs is made possible by the fact that $N C^{(A)}(n)$ and $N C^{(B)}(n)$ embed naturally into the Cayley graphs of the symmetric group $S_{n}$, and respectively of the hyperoctahedral group $W_{n}$ (see discussion in Section 3 below).

When worked out explicitly, $\mathrm{t}^{(B)}$ is an associative binary operation on series with coefficients from $\mathbf{C}^{2}$. The property of $\boldsymbol{\otimes}^{(B)}$ which is important for our investigation is described as follows (see Section 5.3 below):

Let $\mathcal{C}$ be the algebra structure on $\mathbf{C}^{2}$ which is obtained by identifying $\left(\alpha^{\prime}, \alpha^{\prime \prime}\right) \in$ $\mathbf{C}^{2}$ with the $2 \times 2$ matrix $\left[\begin{array}{cc}\alpha^{\prime} & \alpha^{\prime \prime} \\ 0 & \alpha^{\prime}\end{array}\right]$. Then $\boldsymbol{⿴ 囗 十 ~}^{(B)}$ can be viewed as a boxed convolution of type A, but with coefficients in $\mathcal{C}$ :

$$
\star^{(B)}=\otimes_{\mathcal{C}}^{(A)} .
$$

The equality $(I)$ is a consequence of the following simple, but important fact which takes place at the level of lattices of partitions: for every $n \geq 1$, the natural "absolute value map" Abs : $N C^{(B)}(n) \rightarrow N C^{(A)}(n)$ is an $(n+1)$-to-1 cover (see Section 1.4 below). As a result of this, the summations over $N C^{(B)}(n)$ that are involved in the definition of $\star^{(B)}$ can be pushed forward in a controlled way to summations over $N C^{(A)}(n)$, and $(I)$ follows.

The fact that the algebra structure $\mathcal{C}$ on $\mathbf{C}^{2}$ plays a role in considerations about the lattices $N C^{(B)}(n)$ had already been noticed in Reiner's work [16. Theorem 16 in Section 6 of [16] can, in fact, be viewed as a result about $\otimes^{(B)}$ (a characterization of $\star^{(B)}$ on a part of its domain of definition), which relates $\Theta^{(B)}$ to the algebra $\mathcal{C}$. This result could also be used to obtain a derivation of $(I)$, different from the one outlined in the preceding paragraph. 
The developments described above suggest that the non-crossing cumulants of type $\mathrm{B}$ should be $\mathbf{C}^{2}$-valued, and should be defined by the formulas which one would normally use for $\mathcal{C}$-valued cumulants of type $\mathrm{A}$ (with $\mathcal{C}$ the algebra structure appearing in $(I)$ ). When this is done, a suitable concept of non-commutative probability space of type B arises at the same time with the definition of the cumulants of type B. From this point on we can pursue the program suggested at the beginning of the discussion: consider the condition of vanishing of mixed cumulants of type B, and rephrase it in terms of moments, in order to arrive at the definition of what is "free independence of type B" (cf. Section 7.2 below).

As a general comment, we note that the type B structures which come into the discussion seem to always be "superimposed" on their counterparts of type A (rather than being totally new objects). This starts at the level of lattices, and goes all the way to algebras of non-commuting variables, where a "non-commutative probability space of type B" is essentially given by a representation of a noncommutative probability space of type A.

Concerning possible directions for future research: The free probability of type $\mathrm{A}$ is a rich theory, and there are quite a few of its aspects - e.g. the free central limit theorem, the theory of reduced free products, or the Fock space models for free independence - for which it is certainly worth looking for type B analogues. It would also be interesting if connections could be established between free probability of type B and the line of research in non-commutative probability started by Bozejko and Speicher [4] on " $\varphi-\psi$ independence".

Following the introduction, the paper is divided into seven sections.

In the first three sections we go over basic facts about the lattice $N C^{(B)}(n)$ and present the relevant connections between $N C^{(B)}(n)$ and the Cayley graph of the hyperoctahedral group $W_{n}$.

Section 4 provides a brief review of some definitions and basic facts which are commonly used in the combinatorics of free probability (of type A), and for which type B analogues will be developed in Sections 5-7.

Section 5 is devoted to the operations of boxed convolution $\star^{(A)}$ and $\star^{(B)}$ which are obtained by suitably particularizing the concept of "restricted convolution of multiplicative functions" to symmetric and (respectively) hyperoctahedral groups.

The concept of non-crossing cumulant of type B is introduced in Section 6; in the same section we obtain an equivalent (more explicit) description of this concept, and we point out the type B analogue of Voiculescu's R-transform.

Finally, in Section 7 we study the condition of vanishing of mixed cumulants of type B, and we arrive at the analogue of type B for the concept of free independence.

Cross-referencing between sections is done by using the subsection number (e.g. "Proposition 3.3" refers to the unique proposition stated in Section 3.3).

Acknowledgment. The work presented here started from discussions between the three authors at MSRI Berkeley, during the special program in operator algebras in 2000-2001. We gratefully acknowledge the hospitality of MSRI during that period.

\section{Non-Crossing PARtitions of type A And of type B}

1.1. Review of non-crossing partitions of type A. The partition lattice of a finite set $F$ has as elements the partitions of $F$ into disjoint non-empty subsets. The non-empty subsets making up a partition are called the blocks (or classes) of 
the partition. If $p$ is a partition of $F$, and $a, b \in F$, we write $a \sim_{p} b$ to denote that $a$ and $b$ are in the same block of $p$. Partitions are ordered by reverse refinement: $p \leq q$ if $p$ is a refinement of $q$, that is, if every block of $p$ is contained in a block of $q$. This partial order has a maximum element $1_{F}$, which has $F$ as its only block, and a minimum element $0_{F}$, in which every block is a singleton.

Now suppose that $F$ is totally ordered. A partition $p$ of $F$ is said to be noncrossing if whenever $a<b<c<d$ in $F$, and $a \sim_{p} c, b \sim_{p} d$, it follows that $b \sim_{p} c$. The set $N C^{(A)}(F)$ of non-crossing partitions of $F$ is itself a lattice, when considered with the partial order induced from the partition lattice of $F$. The same $1_{F}$ and $0_{F}$ as before serve as maximal and respectively minimal elements of $N C^{(A)}(F)$. When $F$ is the set

$$
[n]:=\{1,2, \ldots, n\},
$$

considered with the usual order, we write $N C^{(A)}(n)$ instead of $N C^{(A)}([n])$. The lattice $N C^{(A)}(n)$ was first studied by Kreweras [11].

One can give a recursive criterion for a partition to be non-crossing: $p$ is a noncrossing partition of $F$ precisely when $p$ has a block $A$ that is an interval in $F$, and $p \backslash\{A\}$ is a non-crossing partition of $F \backslash A$.

A geometric picture of the non-crossing condition is obtained by placing the points of $F$ in order around a circle. Given a partition $p$ of $F$, form for each block of $p$ the convex hull of the block (i.e., the smallest convex set in the plane containing the points of the block). The partition is non-crossing precisely when the convex hulls of different blocks are non-intersecting. This makes it clear that the non-crossing condition is preserved under cyclic permutations of $F$.

$N C^{(A)}(n)$ has a complementation map $\mathrm{Kr}$ (introduced by Kreweras), given as follows. Consider the totally ordered set

$$
J=\{1<\overline{1}<2<\overline{2}<\cdots<n<\bar{n}\} .
$$

For $p \in N C^{(A)}(n)$, its complement $\operatorname{Kr}(p)$ is the largest element $q$ of

$$
N C^{(A)}(\{\overline{1}, \overline{2}, \ldots, \bar{n}\}) \cong N C^{(A)}(n)
$$

such that $p \cup q$ is a non-crossing partition of $J$. Then $\mathrm{Kr}$ is an order-reversing bijection of $N C^{(A)}(n)$. The following property of $\mathrm{Kr}$ is also worth recording:

$$
\operatorname{blno}(p)+\operatorname{blno}(\operatorname{Kr}(p))=n+1, \quad \forall p \in N C^{(A)}(n),
$$

where $\operatorname{blno}(p)$ stands for the number of blocks of the partition $p$.

A left-hand version $\mathrm{Kr}^{\prime}$ of the Kreweras complement has the same description as $\mathrm{Kr}$, but with $J$ replaced by

$$
J^{\prime}=\{\overline{1}<1<\overline{2}<2<\cdots<\bar{n}<n\} .
$$

One has $\mathrm{Kr}^{\prime} \circ \mathrm{Kr}=i d$ on $N C^{(A)}(n)$.

Note that, via suitable identifications, one can talk about $\mathrm{Kr}$ and $\mathrm{Kr}^{\prime}$ on $N C^{(A)}(F)$, where $F$ is any totally ordered set.

1.2. Non-crossing partitions of type B. The type B analogue of the lattice of non-crossing partitions was introduced by Reiner [16]. Consider the totally ordered set

$$
[ \pm n]:=\{1<2<\cdots<n<-1<-2<\cdots<-n\}
$$


with its inversion map $a \mapsto-a$. One defines $N C^{(B)}(n)$ to be the subset of $N C^{(A)}([ \pm n]) \cong N C^{(A)}(2 n)$ consisting of partitions that are invariant under the inversion map.

If $\pi \in N C^{(B)}(n)$, then the blocks of $\pi$ are of two types: those that are inversioninvariant, and those that are not. From the non-crossing condition it is easily seen that $\pi$ can actually have at most one block that is inversion-invariant; if this exists, it will be called the zero-block of $\pi$. The other blocks of $\pi$ must come in pairs: if $X$ is a non-inversion-invariant block, then $-X$ is also a block, different from $X$.

It is immediate that $N C^{(B)}(n)$ is a sublattice of $N C^{(A)}([ \pm n])$ containing the minimal and maximal elements of $N C^{(A)}([ \pm n])$.

Furthermore, it is easily seen that $N C^{(B)}(n)$ is closed under the Kreweras complements $\mathrm{Kr}$ and $\mathrm{Kr}^{\prime}$ considered on $N C^{(A)}([ \pm n])$. When restricted from $N C^{(A)}([ \pm n])$ to $N C^{(B)}(n)$, the maps $\mathrm{Kr}$ and $\mathrm{Kr}^{\prime}$ will, therefore, yield two antiisomorphisms of $N C^{(B)}(n)$, inverse to each other, and which will also be called Kreweras complementation maps (on $N C^{(B)}(n)$ ). Note that for $\pi \in N C^{(B)}(n)$ there is no ambiguity about the meaning of " $\operatorname{Kr}(\pi)$ ", no matter whether $\pi$ is viewed as an element of $N C^{(B)}(n)$ or of $N C^{(A)}([ \pm n])$.

Let us observe that equation (1.2) gives us

$$
\operatorname{blno}(\pi)+\operatorname{blno}(\operatorname{Kr}(\pi))=2 n+1, \quad \forall \pi \in N C^{(B)}(n) .
$$

This has the following consequence: given $\pi \in N C^{(B)}(n)$, exactly one of the two partitions $\pi$ and $\operatorname{Kr}(\pi)$ has a zero-block. Indeed, a partition in $N C^{(B)}(n)$ has a zero-block if and only if it has an odd number of blocks; and (1.4) implies that exactly one of $\pi$ and $\operatorname{Kr}(\pi)$ has an odd number of blocks.

\subsection{Absolute value of a non-crossing partition of type B.}

Notation. Let $n$ be a positive integer, and consider the sets $[n]$ and $[ \pm n]$ appearing in the preceding subsections (cf. (1.1) and (1.3)). We denote by Abs : $[ \pm n] \rightarrow[n]$ the absolute value map sending $\pm i$ to $i$, for $1 \leq i \leq n$. Moreover, if $X$ is a subset of $[ \pm n]$, we will use the notation $\operatorname{Abs}(X)$ for the set $\{\operatorname{Abs}(x): x \in X\} \subset[n]$.

Proposition and Definition. Let $n$ be a positive integer, and let $\pi$ be a partition in $N C^{(B)}(n)$. Then the sets of the form

$$
\operatorname{Abs}(X) \text {, with } X \text { a block of } \pi
$$

form a non-crossing partition $p$ of $[n]$. This $p \in N C^{(A)}(n)$ will be called the absolute value of $\pi$, and will be denoted $\operatorname{Abs}(\pi)$.

Proof. It is clear that $p=\operatorname{Abs}(\pi)$ is a partition of $[n]$. In fact, $a \sim_{p} b \Leftrightarrow\left(a \sim_{\pi}\right.$ $b$ and $\left.-a \sim_{\pi}-b\right)$ or $\left(a \sim_{\pi}-b\right.$ and $\left.-a \sim_{\pi} b\right)$.

We have to check the non-crossing condition for $p$. Suppose that $a<b<c<d$ in $[n]$ and $a \sim_{p} c, b \sim_{p} d$. Then we have $a \sim_{\pi} c$ or $a \sim_{\pi}-c$ in $[ \pm n]$, and $b \sim_{\pi} d$ or $b \sim_{\pi}-d$ in $[ \pm n]$.

Suppose $a \sim_{\pi} c$. If $b \sim_{\pi} d$, then $b \sim_{\pi} c$ by the non-crossing condition for $\pi$; so $b \sim_{p} c$. If $b \sim_{\pi}-d$, then, since $a<b<c<-d$ in $[ \pm n]$, we again have $b \sim_{\pi} c$ by the non-crossing condition for $\pi$; so again $b \sim_{p} c$.

The discussion of the case when $a \sim_{\pi}-c$ is similar, and leads to the same conclusion that $b \sim_{p} c$. 
1.4. The absolute value is an $(n+1)$-to- 1 cover. As counted by Kreweras [11, the number of partitions in $N C^{(A)}(n)$ is a Catalan number,

$$
\operatorname{card}\left(N C^{(A)}(n)\right)=\frac{1}{n+1}\left(\begin{array}{c}
2 n \\
n
\end{array}\right) .
$$

Reiner [16] observes that in the type B case we have simply

$$
\operatorname{card}\left(N C^{(B)}(n)\right)=\left(\begin{array}{c}
2 n \\
n
\end{array}\right)
$$

and that, in fact, several formulas in type B are simpler than their counterparts in type $\mathrm{A}$, because of the absence of the factor $1 /(n+1)$.

In the present paper we will use the following fact, which gives a nice interpretation for the relation between the cardinalities of $N C^{(A)}(n)$ and of $N C^{(B)}(n)$.

Theorem. Let $n$ be a positive integer. Then $\pi \mapsto \operatorname{Abs}(\pi)$ is an $(n+1)$-to-1 map from $N C^{(B)}(n)$ onto $N C^{(A)}(n)$.

We break the argument proving the theorem into several lemmas.

Lemma 1. Let $n$ be a positive integer. Then

$$
\operatorname{Kr}(\operatorname{Abs}(\pi))=\operatorname{Abs}(\operatorname{Kr}(\pi)), \quad \pi \in N C^{(B)}(n) .
$$

Note: On the left-hand side of (1.5), "Kr" denotes a Kreweras complement in $N C^{(A)}(n)$, while on the right-hand side, "Kr" denotes a Kreweras complement in $N C^{(B)}(n)$.

Proof of Lemma 1. Fix $\pi \in N C^{(B)}(n)$, about which we will prove (1.5).

Let us observe that $\operatorname{Abs}(\pi) \cup \operatorname{Abs}(\operatorname{Kr}(\pi))$ is a non-crossing partition of the set $1<\overline{1}<\cdots<n<\bar{n}$. This follows by applying Proposition 1.3 to $\pi \cup \operatorname{Kr}(\pi)$, which is a non-crossing partition of the set

$$
1<\overline{1}<\cdots<n<\bar{n}<-1<-\overline{1}<\cdots<-n<-\bar{n} .
$$

Since $\operatorname{Kr}(\operatorname{Abs}(\pi))$ is maximal with the property that $\operatorname{Abs}(\pi) \cup \operatorname{Kr}(\operatorname{Abs}(\pi))$ is noncrossing, it follows that

$$
\operatorname{Abs}(\operatorname{Kr}(\pi)) \leq \operatorname{Kr}(\operatorname{Abs}(\pi)) .
$$

In order to complete the proof of (1.5), it is sufficient to check that $\operatorname{Abs}(\operatorname{Kr}(\pi))$ and $\operatorname{Kr}(\operatorname{Abs}(\pi))$ have the same number of blocks. From (1.2) we know that $\operatorname{Kr}(\operatorname{Abs}(\pi))$ has $n+1-\operatorname{blno}(\operatorname{Abs}(\pi))$ blocks. On the other hand, when we use (1.4) and take into account that exactly one of $\pi$ and $\operatorname{Kr}(\pi)$ has a zero-block, we obtain that the number of blocks of $\operatorname{Abs}(\operatorname{Kr}(\pi))$ is also equal to $n+1-\operatorname{blno}(\operatorname{Abs}(\pi))$.

Lemma 2. Let $n$ be a positive integer. Suppose that $X, Y, Z$ are non-empty subsets of $[ \pm n]$ such that all of the following hold:

(1) $Z=-Z, X \cap(-X)=\emptyset, Y \cap(-Y)=\emptyset$.

(2) The family of sets $Z, X$ and $-X$ is non-crossing.

(3) The family of sets $Z, Y$ and $-Y$ is non-crossing.

(4) $\operatorname{Abs}(X)=\operatorname{Abs}(Y) \subset[n]$.

Then either $X=Y$, or $X=-Y$. 
Proof of Lemma 2. Fix a $j \in \operatorname{Abs}(X)=\operatorname{Abs}(Y)$. By replacing if necessary $X$ with $-X$ and $Y$ with $-Y$, we can assume without loss of generality that $j \in X \cap Y$. The conclusion of the proof then has to be that $X=Y$.

Draw $1<2<\cdots<n<-1<-2<\cdots<-n$ around a circle, and cut out of the circle the convex hull (boundary included) of the points belonging to $Z$. Thus we cut out a convex $(2 m)$-gon, where $\operatorname{card}(Z)=2 m$, with $m \leq n$; and what remains of the circle is a union of $2 m$ domains, each of them bounded by a side of the $2 m$-gon and by an arc of the circle. Note that none of these $2 m$ domains can contain a pair of points $i$ and $-i$ with $1 \leq i \leq n$. (This is because a line connecting two points drawn around the circle and belonging to the same domain cannot intersect the convex $(2 m)$-gon that was cut out, while the line connecting $i$ and $-i$ does intersect the (2m)-gon.)

Now look at the domain (out of the $2 m$ domains constructed in the preceding paragraph) that contains the point $j$. Let $U \subset[ \pm n]$ be the set of points drawn around the circle, and which belong to that domain. From the hypothesis that $X$ and $Z$ do not cross we obtain that $X \subset U$; similarly, the hypothesis that $Y$ and $Z$ do not cross gives that $Y \subset U$. Finally, the observation made at the end of the preceding paragraph shows that the absolute value function is injective on $U$; therefore, the hypothesis $\operatorname{Abs}(X)=\operatorname{Abs}(Y)$ implies $X=Y$.

Lemma 3. Let $n$ be a positive integer, and let $\pi, \rho$ be in $N C^{(B)}(n)$. Suppose that

(1) $\operatorname{Abs}(\pi)=\operatorname{Abs}(\rho)$, and that

(2) $\pi$ and $\rho$ have a common zero-block $Z=-Z \subset[ \pm n]$.

Then $\pi=\rho$.

Proof of Lemma 3. Let $X$ be a non-zero block of $\pi$. Since $\operatorname{Abs}(\pi)=\operatorname{Abs}(\rho)$, there exists a block $Y$ of $\rho$ such that $\operatorname{Abs}(Y)=\operatorname{Abs}(X) . \quad Y$ is not a zero-block of $\rho$ (because $\rho$ has only one zero-block, which is $Z$, with $\operatorname{Abs}(Z)$ disjoint from $\operatorname{Abs}(X)=$ $\operatorname{Abs}(Y))$. Thus Lemma 2 applies to $X, Y$ and $Z$, and gives us that either $X=Y$ or $X=-Y$. In either case, the pair of blocks $X,-X$ of $\pi$ must coincide with the blocks $Y,-Y$ of $\rho$. In this way we obtain that every block of $\pi$ also is a block of $\rho$, and the conclusion $\pi=\rho$ follows.

Proof of the Theorem. We will prove the inequality

$$
\operatorname{card}\left(\left\{\pi \in N C^{(B)}(n): \operatorname{Abs}(\pi)=p\right\}\right) \leq n+1, \forall p \in N C^{(A)}(n) .
$$

This will imply the statement of the theorem, because we know that

$$
\operatorname{card}\left(N C^{(B)}(n)\right)=(n+1) \cdot \operatorname{card}\left(N C^{(A)}(n)\right) .
$$

We fix $p \in N C^{(A)}(n)$, for which we show that (1.6) holds. We denote $\operatorname{Kr}(p)=: q$. Let $A_{1}, \ldots, A_{k}$ be the list of the blocks of $p$, and let $A_{k+1}, \ldots, A_{n+1}$ be the list of the blocks of $q$; this notation can be used because we know that the total number of blocks of $p$ and $q$ is $n+1$ (cf. (1.2)).

Let $\pi \in N C^{(B)}(n)$ be such that $\operatorname{Abs}(\pi)=p$. Then we have $\operatorname{Abs}(\operatorname{Kr}(\pi))=q$, by Lemma 1. Exactly one of $\pi$ and $\operatorname{Kr}(\pi)$ has a zero-block $Z=-Z \subset[ \pm n]$. If $\pi$ has a zero-block $Z$, then $\operatorname{Abs}(Z)=A_{m}$ for some $1 \leq m \leq k$; while if $\operatorname{Kr}(\pi)$ has a zero-block $Z$, then $\operatorname{Abs}(Z)=A_{m}$ for some $k<m \leq n+1$. In either case, we end by assigning to $\pi$ a number $m \in\{1, \ldots, n+1\}$, determined by the equality $\operatorname{Abs}(Z)=A_{m}$. 
In this way we get a function

$$
\Phi:\left\{\pi \in N C^{(B)}(n): \operatorname{Abs}(\pi)=p\right\} \rightarrow\{1, \ldots, n+1\}
$$

defined by setting $\Phi(\pi):=m$, where $m$ is obtained from $\pi$ in the way described in the preceding paragraph.

But now, from Lemma 3 it follows that the function $\Phi$ defined above is injective. Indeed, let $\pi, \rho$ be in the domain of $\Phi$ (i.e., they are partitions in $N C^{(B)}(n)$ such that $\operatorname{Abs}(\pi)=\operatorname{Abs}(\rho)=p)$, and suppose that $\Phi(\pi)=\Phi(\rho)=m \in\{1, \ldots, n+1\}$. If $m \leq k$, then Lemma 3 applies directly to give $\pi=\rho$. If $m \geq k+1$, then Lemma 3 applies to give $\operatorname{Kr}(\pi)=\operatorname{Kr}(\rho)$ - but this still implies that $\pi=\rho$, since $\mathrm{Kr}$ is one-to-one on $N C^{(B)}(n)$.

Finally, since the function $\Phi$ of (1.7) is injective, its domain can have at most $n+1$ elements; this is exactly (1.6).

Remark. The proof of the theorem actually tells us how to concretely construct the partitions $\pi \in \operatorname{Abs}^{-1}(p) \subset N C^{(B)}(n)$, for a given $p \in N C^{(A)}(n)$. First we choose a block of either $p$ or $\operatorname{Kr}(p)$, which is to be "lifted into a zero-block" (of either $\pi$ or $\operatorname{Kr}(\pi))$. Then all the blocks of $\pi$ are completely determined by the fact they must have blocks of $p$ as absolute values, and that they cannot cross the chosen zero-block.

For instance, suppose that $p=\{(1,2),(3,4)\} \in N C^{(A)}(4)$. We have $\operatorname{Kr}(p)=$ $\{(1),(2,4),(3)\}$. If we e.g. choose the block $(1,2)$ of $p$ as the one to be lifted into a zero-block, then we get the partition $\pi=\{(1,2,-1,-2),(3,4),(-3,-4)\} \in$ $\operatorname{Abs}^{-1}(p)$, while if we choose the block (1) of $\operatorname{Kr}(p)$ as the one to be lifted into a zero-block, then we get a partition $\pi \in \operatorname{Abs}^{-1}(p)$ that has $\operatorname{Kr}(\pi)=\{(1,-1)$, $(2,4),(-2,-4),(3),(-3)\}$, and that must, therefore, be $\pi=\{(1,-2),(-1,2),(3,4)$, $(-3,-4)\}$.

\section{The "CAYley GRAPH" FRAMEWORK}

2.1. Marked groups. We consider objects of the form $(G, T)$, where $G$ is a group and $T$ is a finite set of generators of $G$. Such a pair $(G, T)$ is sometimes called "a marked group". We will assume that $T$ does not contain the unit $e$ of $G$, and more importantly that:

(i) $T$ is closed under taking the inverse $\left(x \in T \Rightarrow x^{-1} \in T\right)$; and

(ii) $T$ is closed under conjugation $\left(x \in T, c \in G \Rightarrow c^{-1} x c \in T\right)$.

2.2. Word-length and distance on a marked group. Let $(G, T)$ be as in Section 2.1. For every element $e \neq a \in G$ we define its length $|a|$ as the smallest positive integer $n$ with the property that $a$ can be written as $a=x_{1} \cdots x_{n}$ with $x_{1}, \ldots, x_{n} \in T$. By convention, the length of $e$ is $|e|:=0$.

It is well-known and easy to prove that the length function $|\cdot|: G \rightarrow\{0,1,2, \ldots\}$ has the following properties:

$$
\begin{cases}|a b| \leq|a|+|b|, & \forall a, b \in G, \\ \left|a^{-1}\right|=|a|, & \forall a \in G, \\ \left|c^{-1} a c\right|=|a|, & \forall a, c \in G .\end{cases}
$$


As a consequence, it is immediate that the formula

$$
d(a, b):=\left|a^{-1} b\right|=\left|b a^{-1}\right|=\left|b^{-1} a\right|=\left|a b^{-1}\right|
$$

makes sense and defines a distance on $G$. Moreover, the distance $d$ is invariant under left and right translations on $G$ (i.e., $d\left(c^{\prime} a c^{\prime \prime}, c^{\prime} b c^{\prime \prime}\right)=d(a, b)$ for all $\left.a, b, c^{\prime}, c^{\prime \prime} \in G\right)$.

2.3. The partial order on a marked group. Let $(G, T)$ be a marked group as in Section 2.1. We introduce a partial order on $G$ by declaring that for $a, b \in G$ we have

$$
a \leq b \stackrel{\text { def }}{\Leftrightarrow} d(e, a)+d(a, b)=d(e, b)
$$

(where $e$ is the unit of $G$, and $d$ is the distance on $G$, as in Section 2.2). It is immediately checked that the prescription (2.3) indeed defines a partial order on $G$, which has the unit $e$ as (unique) minimal element. It is also clear that for $a, b \in G$ we have the implication

$$
a \leq b \Rightarrow|a| \leq|b|,
$$

the converse of which is not generally true.

For any $a, b \in G$ such that $a \leq b$ we will use the natural interval notation

$$
[a, b]:=\{c \in G: a \leq c \leq b\} .
$$

When trying to understand the partial order on $G$, a useful concept is that of "cover". For $a, b \in G$ we say that $b$ covers $a$ if $a \leq b$ and there are no elements of $G$ properly sitting between $a$ and $b$ (i.e., if the interval $[a, b]$ is reduced to just $a$ and $b$ ). As is easily checked, an equivalent description for the fact that $b$ covers $a$ is " $a \leq b$ and $|b|=|a|+1$ ".

2.4. Cayley graphs. Let $(G, T)$ be a marked group as in Section 2.1. The Cayley graph of $(G, T)$ is a graph whose vertices are the elements of $G$, and whose edges are the two-element subsets $\{a, b\} \subset G$ such that $d(a, b)=1$ (with $d$ defined as in $(2.2))$.

The distance and the partial order on $G$, as discussed in Sections 2.2 and 2.3, have natural interpretations in the Cayley graph of $(G, T)$. For instance, for $a, b \in G$, the prescription used in (2.3) to define what $a \leq b$ means can now be interpreted as saying that " $a$ lies on a geodesic from $e$ to $b$, in the Cayley graph of $(G, T)$ ".

Cayley graphs are generally considered for marked groups $(G, T)$ with fewer conditions imposed on $T$ than we had in Section 2.1 (see, e.g., Chapter IV of [8]). For our purposes the framework of Section 2.1 is nevertheless appropriate, because we will only focus on the following two classes of examples.

2.5. Example: the symmetric groups. Let $n \geq 2$ be an integer, and let $S_{n}$ denote the symmetric group on the set $[n]$ (i.e., the group of all permutations of $[n]=\{1, \ldots, n\})$. The permutations $t \in S_{n}$ will usually be written in cycle notation. (For example, $t=(1,3,4)(2,6)(5) \in S_{6}$ is the permutation $\left(\begin{array}{cccccc}1 & 2 & 3 & 4 & 5 & 6 \\ 3 & 6 & 4 & 1 & 5 & 2\end{array}\right)$, which partitions the set $\{1, \ldots, 6\}$ into three orbits, and was therefore written as a product of 3 cycles. One often omits the cycles corresponding to orbits of cardinality 1 ; thus the same $t \in S_{6}$ may also appear written as $t=(1,3,4)(2,6)$.) The term "long cycle" is used for a permutation $t \in S_{n}$ that has only one orbit, necessarily equal to $[n]$. 
Let $T_{n}$ be the set of all transpositions in $S_{n}$ (i.e., the set of permutations in $S_{n}$ that have one orbit of length 2 and $n-2$ orbits of length 1$)$. It is clear that $\left(S_{n}, T_{n}\right)$ is a marked group, satisfying all the conditions discussed in Section 2.1. The length function for $\left(S_{n}, T_{n}\right)$ is described by the formula

$$
|t|=n-(\# \text { of orbits of } t), \quad t \in S_{n},
$$

as is easily checked.

We remark that these are not the usual (Coxeter) generating set and length function for the symmetric groups; the usual generating set consists of adjacent transpositions $(i, i+1)$ only, and is not invariant under conjugation. See, for example, 9.

Concerning the partial order on $S_{n}$ (defined as in Section 2.3), it is worth pointing out how the concept of cover is explicitly described in this example. It is easily checked that for $t_{1}, t_{2} \in S_{n}$ we have

$$
t_{2} \text { covers } t_{1} \Leftrightarrow\left\{\begin{array}{l}
t_{2}=t_{1} r, \text { where } r=(i, j) \in T_{n} \text { is such that } \\
i \text { and } j \text { belong to different orbits of } t_{1} .
\end{array}\right.
$$

The effect of the right multiplication by $r$ in the equality $t_{2}=t_{1} r$ of (2.7) is that the two orbits of $t_{1}$ that contain $i$ and $j$ are united into one orbit of $t_{2}$ (which thus contains both $i$ and $j$ ).

2.6. Example: the hyperoctahedral groups. Let $n$ be a positive integer, and let $W_{n}$ denote the hyperoctahedral group with $2^{n} n$ ! elements (or in other words, the Weyl group of type $\mathrm{B}_{n}$ ). The realization of $W_{n}$ which we will use in the present paper is as the group of permutations $\tau$ of the set $[ \pm n]$ (with $[ \pm n]$ as defined in (1.3)), which have the property that

$$
\tau(-i)=-\tau(i), \quad 1 \leq i \leq n .
$$

Hence, we will view $W_{n}$ as a subgroup of $S_{ \pm n}$, the symmetric group on $[ \pm n]$.

Every $\tau \in W_{n}$ decomposes as a product of cycles (since $\tau$ is in particular an element of $S_{ \pm n}$ ). Because of (2.8), we see that the cycle decomposition of $\tau$ may contain two kinds of cycles: some of which are inversion invariant, and some of which are not. The cycles of the non-invariant kind must come in pairs (e.g. if $(1,2,-6,3)$ is a cycle of $\tau$, then $(-1,-2,6,-3)$ must also be a cycle of $\tau)$.

As generating set for $W_{n}$ we take the set of reflections $R_{n}$ which consists of all transpositions $(i,-i), 1 \leq i \leq n$, and of all the products of two transpositions $(i, j)(-i,-j)$ with $i, j \in[ \pm n]$ and $|i| \neq|j|$. Then $\left(W_{n}, R_{n}\right)$ is a marked group, and $R_{n}$ has all the properties considered in Section 2.1. The length function for $\left(W_{n}, R_{n}\right)$ is described by the formula:

$$
|\tau|=n-\left(\begin{array}{c}
\# \text { of pairs of orbits of } \tau \\
\text { that are non-invariant under inversion }
\end{array}\right), \quad \tau \in W_{n} .
$$

Again, these are not the usual (Coxeter) generating set and length function on the hyperoctahedral groups; the usual generating set consists of the adjacent reflections $(i, i+1)(-i,-i-1)$ (for $1 \leq i \leq n-1)$ and the transposition $(1,-1)$ only, and is not invariant under conjugation.

Continuing the analogy with the preceding example, let us now pass to the partial order on $W_{n}$ (defined as in Section 2.3), and let us look at the explicit description for the concept of cover with respect to this partial order. The description is more 
complicated than what we had in Example 2.5, but the reader should have no difficulty in checking that it is done as follows:

Lemma. Let $\tau_{1}, \tau_{2}$ be in $W_{n}$. Then $\tau_{2}$ covers $\tau_{1}$ in the partial order coming from $\left(W_{n}, R_{n}\right)$ if and only if $\rho:=\tau_{1}^{-1} \tau_{2}$ is in $R_{n}$ and falls in one of the following four situations:

(a) $\rho=(i,-i)$, where $i$ and $-i$ belong to different orbits of $\tau_{1}$.

(b) $\rho=(i, j)(-i,-j)$ with $|i| \neq|j|$ and where $i$ and $-i$ belong to the same orbit of $\tau_{1}$, but $j$ and $-j$ do not belong to the same orbit of $\tau_{1}$.

(c) $\rho=(i, j)(-i,-j)$ with $|i| \neq|j|$ and where no two of $i, j,-i,-j$ belong to the same orbit of $\tau_{1}$.

(d) $\rho=(i, j)(-i,-j)$ with $|i| \neq|j|$, where $i$ and $-j$ belong to the same orbit of $\tau_{1}$, and this orbit is not invariant under inversion (and hence does not contain $-i$ and $j$ ).

In the situations (a), (b), (c) of the preceding lemma, the effect of the right multiplication with $\rho$ in " $\tau_{2}=\tau_{1} \rho$ " is that some distinct orbits of $\tau_{1}$ are united to form larger orbits of $\tau_{2}$. The situation (d) is different; in this case, if $X \subset[ \pm n]$ denotes the orbit of $\tau_{1}$ that contains $i$ and $-j$, then right multiplication by $\rho$ has the effect of replacing the orbits $X$ and $-X$ of $\tau_{1}(X \neq-X)$ by two inversion invariant orbits $Y$ and $Z$ of $\tau_{2}$ such that $Y \cup Z=X \cup(-X), i,-i \in Y$ and $j,-j \in Z$.

An immediate consequence of the lemma is the following.

Corollary. If $\tau_{1} \leq \tau_{2}$ in $W_{n}$, then $\tau_{2}$ has at least as many inversion-invariant orbits as $\tau_{1}$.

Proof. Without loss of generality we may assume that $\tau_{2} \operatorname{covers} \tau_{1}$. Then $\rho:=\tau_{1}^{-1} \tau_{2}$ must fall in one of the four situations (a)-(d) described in the preceding lemma. Let $N_{1}$ and $N_{2}$ denote the number of inversion-invariant orbits of $\tau_{1}$ and of $\tau_{2}$, respectively. By direct inspection we see that $N_{2}=N_{1}$ in situations (b) and (c), $N_{2}=N_{1}+1$ in situation (a), and $N_{2}=N_{1}+2$ in situation (d). Hence, the inequality $N_{1} \leq N_{2}$ always holds.

2.7. Restricted convolution. The last ingredient of the "Cayley graph framework" which we want to consider is a convolution operation for complex-valued functions defined on the corresponding group.

Definition. Let $(G, T)$ be a marked group as in Section 2.1, and let $\mathcal{F}(G, \mathbf{C})$ denote the set of all complex-valued functions on $G$. On $\mathcal{F}(G, \mathbf{C})$ we define an operation of restricted convolution $*_{r}$ via the following formula:

$$
\left(u *_{r} v\right)(a)=\sum_{\substack{b, c \in G, b c=a,|b|+|c|=|a|}} u(b) v(c) .
$$

Remarks.

(1) On the right-hand side of equation (2.10) we are dealing with a finite sum, because $T$ is assumed to be finite (and thus for every $k \geq 0$ there are at most $(\operatorname{card}(T))^{k}$ elements $a \in G$ such that $\left.|a|=k\right)$.

(2) Referring to the partial order in a marked group, we could also write (2.10) in the form

$$
\left(u *_{r} v\right)(a)=\sum_{b \in[e, a]} u(b) v\left(b^{-1} a\right) .
$$


(3) While (2.10) and (2.11) are indeed reminiscent of the convolution operation on $G$, the condition $|b|+|c|=|a|$ required in the sum on the right-hand side of (2.10) changes things quite a bit in comparison to the unrestricted convolution. For instance it is immediate that $\left(u *_{r} v\right)(e)=u(e) v(e)$, or that $\left(u *_{r} v\right)(x)=$ $u(e) v(x)+u(x) v(e)$ for $x \in T$.

We leave it as an exercise to the reader to make the straightforward verifications proving the following proposition.

Proposition. Let $(G, T)$ be a marked group. Then $\mathcal{F}(G, \mathbf{C})$ is a unital complex algebra with the usual (pointwise) vector space operations and with the restricted convolution $*_{r}$ as multiplication. The unit of $\mathcal{F}(G, \mathbf{C})$ is the characteristic function $\chi_{e}$ of the unit of $G\left(\chi_{e}(e)=1\right.$, and $\chi_{e}(a)=0$ for $a \neq e$ in $\left.G\right)$.

\section{Non-Crossing PARTitions AND CAYley GRAPHS}

3.1. $N C^{(A)}(n)$ and the symmetric group $S_{n}$. Consider again the marked group $\left(S_{n}, T_{n}\right)$ of Section 2.5] We will look at the interval $[e, c]$ in the corresponding partial order on $S_{n}$, where $e$ is the unit of $S_{n}$ and $c$ is the "forward" long cycle $c=(1,2, \ldots, n)$ on $[n]$. It was observed by one of us in 3 that this interval provides a "group-theoretic incarnation" of $N C^{(A)}(n)$. More precisely, we have

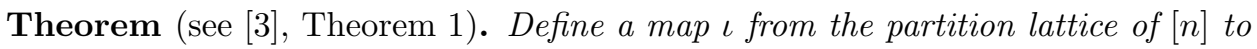
$S_{n}$, as follows:

- First define $\iota$ on the set of subsets of $[n]$ by $\iota\left(\left\{a_{1}, a_{2}, \ldots, a_{k}\right\}\right)=\left(a_{1}, a_{2}, \ldots, a_{k}\right)$, if $a_{1}<a_{2}<\cdots<a_{k}$;

- Extend $\iota$ to partitions of $[n]$ by defining $\iota$ of a partition to be the product of $\iota(F)$, where $F$ runs over the blocks of the partition. (The product is well defined since the cycles $\iota(F)$ are disjoint and, hence, mutually commuting.)

Then the restriction of $\iota$ to $N C^{(A)}(n)$ has range equal to the interval $[e, c] \subset S_{n}$, and $\iota$ is an order isomorphism between $N C^{(A)}(n)$ and the interval $[e, c]$ in $S_{n}$.

Remark. The Kreweras complements $\mathrm{Kr}, \mathrm{Kr}^{\prime}$ reviewed in Section 1.1 have an interesting interpretation in terms of the poset isomorphism $\iota: N C^{(A)}(n) \rightarrow[e, c]$. One has

$$
\iota(\operatorname{Kr}(p))=\iota(p)^{-1} c \text { and } \iota\left(\operatorname{Kr}^{\prime}(p)\right)=c \iota(p)^{-1}, \quad \forall p \in N C^{(A)}(n) .
$$

It actually turns out that the order structure of every subinterval $[e, b] \subset[e, c]$ is closely related to the lattices of non-crossing partitions. This comes as a consequence of some basic properties of the partial order on $S_{n}$, which were put into evidence in 2 and are reviewed in the following proposition.

Proposition (see Section 1.2 in [2]).

(1) Suppose that $a \in S_{n}$ has word-length $|a|=k$, and that $a=t_{1} \cdots t_{k}$ is an expression for an element $a$ as a product of $k$ transpositions. Then for every $1 \leq j \leq k$, the two elements of $[n]$ that are transposed by $t_{j}$ belong to the same orbit of $a$.

(2) If $a \leq b$ in $S_{n}$, then every orbit of $a$ is contained in an orbit of $b$ (and as a consequence, every fixed point of $b$ must also be a fixed point of a).

(3) Let $b \neq e$ be a permutation in $S_{n}$, let $F_{1}, \ldots, F_{k} \subset[n]$ be the orbits of $b$ that contain more than one element, and let $b=b_{1} \cdots b_{k}$ be the corresponding factorization of $b$ into cycles (for every $1 \leq j \leq k, b_{j}$ acts like $b$ on $F_{j}$ and acts 
like e on $\left.[n] \backslash F_{j}\right)$. If $a_{1} \in\left[e, b_{1}\right], \ldots, a_{k} \in\left[e, b_{k}\right]$, then the permutations $a_{1}, \ldots, a_{k}$ commute with each other, and $a:=a_{1} \cdots a_{k}$ is in $[e, b]$.

(4) Let $e \neq b \in S_{n}$ and its factorization $b=b_{1} \cdots b_{k}$ be as in (3). Then every permutation $a \in[e, b]$ can be uniquely written in the form $a=a_{1} \cdots a_{k}$, where $a_{1} \in\left[e, b_{1}\right], \ldots, a_{k} \in\left[e, b_{k}\right]$.

Suppose now that $e \neq b \leq c$ (where $c=(1,2, \ldots, n)$ as before) and that $b$ is factored as $b=b_{1} \cdots b_{k}$ as in statements (3) and (4) of the proposition. Then the proposition tells us that we have a canonical poset isomorphism

$$
[e, b] \cong\left[e, b_{1}\right] \times \cdots \times\left[e, b_{k}\right] .
$$

Moreover, let us fix a $j, 1 \leq j \leq k$, and let us denote by $F_{j}$ the unique orbit of $b_{j}$ that is not reduced to one point. Then the interval $\left[e, b_{j}\right]$ consists of permutations $a \in S_{n}$ such that $a$ fixes every element of $[n] \backslash F_{j}$, and such that we have $a \mid F_{j} \leq$ $b_{j} \mid F_{j}\left(=b \mid F_{j}\right)$, where the latter inequality is considered in the appropriate marked group $\left(S_{F_{j}}, T_{F_{j}}\right)$. Since $b \mid F_{j}$ is a long cycle on $F_{j}$, the theorem presented above in this subsection gives us that $\left[e, b_{j}\right] \cong N C^{(A)}\left(F_{j}\right)$.

So in conclusion, for $e \neq b \leq c$, we obtain

$$
\begin{aligned}
{[e, b] } & \cong N C^{(A)}\left(F_{1}\right) \times \cdots \times N C^{(A)}\left(F_{k}\right) \\
& \cong N C^{(A)}\left(l_{1}\right) \times \cdots \times N C^{(A)}\left(l_{k}\right),
\end{aligned}
$$

where $F_{1}, \ldots, F_{k}$ are the orbits of $b$ consisting of more than one element, and where $l_{j}$ is the cardinality of $F_{j}, 1 \leq j \leq k$.

Since (due to the theorem presented above) $[e, b]$ is naturally identified to a subinterval of $N C^{(A)}(n)$, the isomorphism (3.3) is closely related to some of the "canonical factorizations" for intervals of $N C^{(A)}(n)$ which are studied in Section 3 of [17].

Other references: the results stated in the above theorem and proposition have recently re-emerged in work concerning the braid groups - see Sections 2, 3 of [ 5 ] and Section 4.1 of [1].

3.2. $N C^{(B)}(n)$ and the hyperoctahedral group $W_{n}$. We now take on the type $\mathrm{B}$ analogues for the facts presented in Section 3.1. We will use the Cayley graph framework of Section 2, particularized to the case of the marked group $\left(W_{n}, R_{n}\right)$ which appears in Section 2.6. We will denote by $\omega$ the long inversion-invariant cycle

$$
\omega:=(1,2, \ldots, n,-1,-2, \ldots,-n) \in W_{n},
$$

and we denote the unit of $W_{n}$ by $\varepsilon$. In this subsection we will look at the interval $[\varepsilon, \omega] \in W_{n}$, considered with respect to the partial order coming from $\left(W_{n}, R_{n}\right)$.

Observe that, since $N C^{(B)}(n) \subset N C^{(A)}([ \pm n])$, the map $\iota: N C^{(A)}([ \pm n]) \rightarrow S_{ \pm n}$ (defined as in Theorem 3.1) gives us by restriction a map $\iota: N C^{(B)}(n) \rightarrow S_{ \pm n}$. In fact, it is clear from the definitions that $\iota\left(N C^{(B)}(n)\right) \subset W_{n} \subset S_{ \pm n}$.

We next state the type B analogue for Theorem 3.1. As it turns out, this result has appeared independently in recent work by several people studying Artin groups of finite type - see Theorem 4.9 of [6], or Section 4.2 of [1].

Theorem. The map $\iota: N C^{(B)}(n) \rightarrow W_{n}$ defined above is an order isomorphism of $N C^{(B)}(n)$ onto the interval $[\varepsilon, \omega] \subset W_{n}$ (where the partial order on $W_{n}$ is as described in Sections 2.3 and 2.6). 
This theorem is actually a fairly straightforward consequence of its sibling of type A (Theorem 3.1), applied to the marked group $\left(S_{ \pm n}, T_{ \pm n}\right)$, where $T_{ \pm n}$ denotes the set of all transpositions in $S_{ \pm n}$. For the reader's convenience we sketch the proof. The main point is to observe the following fact:

Lemma. If $\tau_{1}, \tau_{2} \in W_{n}$, and $\tau_{1} \leq \tau_{2} \leq \omega$ with respect to the partial order coming from $\left(W_{n}, R_{n}\right)$, then $\tau_{1} \leq \tau_{2}$ with respect to the partial order coming from $\left(S_{ \pm n}, T_{ \pm n}\right)$.

Proof of the Lemma. Observe first that any $\tau \in[\varepsilon, \omega] \subset W_{n}$ has at most one inversion-invariant orbit. This follows from Corollary 2.6 and the fact that $\omega$ has one inversion-invariant orbit.

We will prove the following implication (which clearly entails the statement of the lemma): If $\tau_{1}, \tau_{2} \in[\varepsilon, \omega]$ are such that $\tau_{2}$ covers $\tau_{1}$ with respect to the order coming from $\left(W_{n}, R_{n}\right)$, then we must have $\tau_{1} \leq \tau_{2}$ with respect to the order coming from $\left(S_{ \pm n}, T_{ \pm n}\right)$.

So let us fix $\tau_{1}, \tau_{2} \in[\varepsilon, \omega]$ such that $\tau_{2}$ covers $\tau_{1}$ with respect to the order coming from $\left(W_{n}, R_{n}\right)$. Then $\rho:=\tau_{1}^{-1} \tau_{2}$ must fall in one of the four situations described in Lemma 2.6. Note that, in fact, $\rho$ cannot fall in situation (d) of that lemma; indeed (as pointed out in the discussion following Lemma 2.6), if $\rho$ were in situation (d), then $\tau_{2}$ would have at least two inversion-invariant orbits, in contradiction to the observation made at the beginning of this proof.

Hence, we have $\tau_{2}=\tau_{1} \rho$, with $\rho$ in one of the situations (a), (b), or (c) described in Lemma 2.6. By comparing these situations (a), (b), (c) with the equivalence stated in (2.7) of Section 2.5, it is immediately seen that in all three cases we indeed have $\tau_{1} \leq \tau_{2}$ with respect to the partial order coming from $\left(S_{ \pm n}, T_{ \pm n}\right)$.

Sketch of Proof of the Theorem. First we have to show that $\iota(\pi) \in[\varepsilon, \omega], \forall \pi \in$ $N C^{(B)}(n)$. This is a consequence of the existence of the Kreweras complement $\operatorname{Kr}(\pi) \in N C^{(B)}(n)$. Indeed, on one hand we know that $\iota(\pi) \iota(\operatorname{Kr}(\pi))=\omega$; this follows from (3.1), with $\pi$ viewed as an element of $N C^{(A)}([ \pm n])$ (recall that, as pointed out in Section 1.2, $\operatorname{Kr}(\pi)$ " has the same meaning when $\pi$ is viewed in $N C^{(B)}(n)$ or in $\left.N C^{(A)}([ \pm n])\right)$. On the other hand, it is straightforward to check, directly from equation (2.9) of Section 2.6, that $|\iota(\pi)|+|\iota(\operatorname{Kr}(\pi))|=n=|\omega|$ (when making this verification it is useful to keep in mind that, as observed in Section 1.2 , exactly one of $\pi$ and $\operatorname{Kr}(\pi)$ has a zero-block). So altogether we find that $\mid\left(\iota(\pi)|+| \iota\left(\pi^{-1} \omega\right)|=| \omega \mid\right.$, and it follows that $\iota(\pi) \leq \omega$.

Next we have to pick an element $\sigma \in[\varepsilon, \omega]$, and show that there exists $\pi \in$ $N C^{(B)}(n)$ such that $\iota(\pi)=\sigma$. We can argue like this: the preceding lemma gives us that $\sigma \leq \omega$ with respect to the order coming from $\left(S_{ \pm n}, T_{ \pm n}\right)$, and, therefore, by Theorem 3.1, there exists $\pi \in N C^{(A)}([ \pm n])$ such that $\iota(\pi)=\sigma$. Moreover, since the blocks of $\pi$ are the orbits of $\iota(\pi)$, we see that $\pi \in N C^{(B)}(n)$.

We next pick partitions $\pi_{1} \leq \pi_{2}$ in $N C^{(B)}(n)$, and we show that $\iota\left(\pi_{1}\right) \leq \iota\left(\pi_{2}\right)$ in $W_{n}$, with respect to the order coming from $\left(W_{n}, R_{n}\right)$. Without loss of generality, we can assume that $\pi_{2}$ covers $\pi_{1}$ in $N C^{(B)}(n)$, i.e., that there are no elements of $N C^{(B)}(n)$ properly between $\pi_{1}$ and $\pi_{2}$. A straightforward inspection (also helped by Proposition 2 in [16]) shows that $\pi_{1}$ and $\pi_{2}$ must fall in one of the following three situations:

(a) $\pi_{1}$ has no inversion-invariant block, and $\pi_{2}$ is obtained from $\pi_{1}$ by merging a block and its inversion to form an inversion-invariant block. 
(b) $\pi_{2}$ is obtained from $\pi_{1}$ by merging the inversion-invariant block of $\pi_{1}$ with a pair of non-inversion-invariant blocks.

(c) $\pi_{2}$ is obtained from $\pi_{1}$ by merging two non-inversion-invariant blocks, as well as merging the inversions of these two blocks.

As is easily checked, the three situations listed above correspond exactly (and in the same order) to the situations (a), (b), (c) described in Lemma 2.6, and applied to the permutations $\iota\left(\pi_{1}\right)$ and $\iota\left(\pi_{2}\right)$. So in all three situations we obtain that indeed $\iota\left(\pi_{1}\right) \leq \iota\left(\pi_{2}\right)$ (and, in fact even more, that $\iota\left(\pi_{2}\right)$ covers $\iota\left(\pi_{1}\right)$ in the partial order coming from $\left.\left(W_{n}, R_{n}\right)\right)$.

Finally, we have to show that if $\pi_{1}, \pi_{2} \in N C^{(B)}(n)$ and $\iota\left(\pi_{1}\right) \leq \iota\left(\pi_{2}\right)$ in $\left(W_{n}, R_{n}\right)$, then $\pi_{1} \leq \pi_{2}$ in $N C^{(B)}(n)$. Here again we first invoke the preceding lemma to obtain that $\iota\left(\pi_{1}\right) \leq \iota\left(\pi_{2}\right)$ in the partial order coming from $\left(S_{ \pm n}, T_{ \pm n}\right)$, and then use Theorem 3.1 to conclude that $\pi_{1}$ is a refinement of $\pi_{2}$.

The bulk of this subsection was devoted to the interval $[\varepsilon, \omega] \subset W_{n}$, where $\omega:=$ $(1,2, \ldots, n,-1,-2, \ldots,-n)$ is a long cycle of invariant type. Let us conclude with a quick look at the interval $[\varepsilon, \gamma] \subset W_{n}$, where $\gamma:=(1,2, \ldots, n)(-1,-2, \ldots,-n) \in$ $W_{n}$ is "a long cycle of non-invariant type".

Proposition. For $\gamma=(1,2, \ldots, n)(-1,-2, \ldots,-n) \in W_{n}$, we have that $[\varepsilon, \gamma] \cong$ $N C^{(A)}(n)$. A natural poset isomorphism from $N C^{(A)}(n)$ onto $[\varepsilon, \gamma] \subset W_{n}$ is obtained as follows: Let $\iota_{o}: N C^{(A)}(n) \rightarrow N C^{(B)}(n)$ be the map that associates to a partition $p=\left\{F_{1}, \ldots, F_{k}\right\} \in N C^{(A)}(n)$ the partition $\iota_{o}(p):=\left\{F_{1}, \ldots, F_{k},-F_{1}, \ldots\right.$, $\left.-F_{k}\right\} \in N C^{(B)}(n)$. Let $\iota: N C^{(B)}(n) \rightarrow W_{n}$ be the map defined at the beginning of this subsection. Then the range of $\iota \circ \iota_{o}: N C^{(A)}(n) \rightarrow W_{n}$ is $[\varepsilon, \gamma] \subset W_{n}$, and $\iota \circ \iota_{o}$ induces a poset isomorphism between $N C^{(A)}(n)$ and $[\varepsilon, \gamma]$.

Proof. Note that $\gamma \leq \omega$ (since $\omega=\gamma \cdot(n,-n)$, and this is a covering situation as in (a) of Lemma 2.6). Hence, $[\varepsilon, \gamma] \subset[\varepsilon, \omega]$, and the inverse of the poset isomorphism $\iota: N C^{(B)}(n) \rightarrow[\varepsilon, \omega]$ provided by the preceding theorem will identify $[\varepsilon, \gamma]$ with the interval:

$$
\begin{aligned}
& {[\{(1),(2), \ldots,(n),(-1),(-2), \ldots,(-n)\},\{(1,2, \ldots, n),(-1,-2, \ldots,-n)\}]} \\
& \quad \subset N C^{(B)}(n) .
\end{aligned}
$$

But the latter interval is precisely $\iota_{o}\left(N C^{(A)}(n)\right)$.

3.3. $N C^{(B)}(n)$ and the hyperoctahedral group $W_{n}$ (continued). In the same setting as in Section 3.2, we will now establish the type B analogue for the facts collected in Proposition 3.1, and for the isomorphism stated (in a type A setting) in equation (3.3) of Section 3.1.

Notation. Let $\tau$ be in $W_{n}, \tau \neq \varepsilon$. Let

$$
X_{1},-X_{1}, \ldots, X_{p},-X_{p}, Z_{1}, \ldots, Z_{q}
$$

be the list of distinct orbits of $\tau$ that have more than one element, where $p, q \geq 0$, and where $Z_{j}=-Z_{j}$ for $j \leq q$. Denote

$$
Y_{1}=X_{1} \cup\left(-X_{1}\right), \ldots, Y_{p}=X_{p} \cup\left(-X_{p}\right), Y_{p+1}=Z_{1}, \ldots, Y_{p+q}=Z_{q},
$$

and for $1 \leq j \leq p+q$ let $\tau_{j}$ be the permutation in $W_{n}$ that acts like $\tau$ on $Y_{j}$ and like $\varepsilon$ on $[ \pm n] \backslash Y_{j}$. Then the writing $\tau=\tau_{1} \cdots \tau_{p+q}$ (with commuting factors $\left.\tau_{1}, \ldots, \tau_{p+q}\right)$ will be called "the cycle factorization of type B" for $\tau$. 
In what follows we will focus on the situation when $\tau \in[\varepsilon, \omega]$, where $\omega$ is the long cycle appearing in Section 3.2 (cf. equation (3.4)). Note that, as a consequence of Corollary 2.6, a permutation $\tau$ in $[\varepsilon, \omega]$ can have at most one inversion-invariant orbit; i.e., for such a $\tau$ the parameter $q$ appearing in the preceding notation is either 0 or 1 .

\section{Proposition.}

(1) Suppose that $\sigma \in[\varepsilon, \omega] \subset W_{n}$ has word-length $|\sigma|=k$, and that $\sigma$ can be written as $\sigma=\rho_{1} \cdots \rho_{k}$ with $\rho_{1}, \ldots, \rho_{k} \in R_{n}$. Then for every $1 \leq j \leq k$, any two elements of $[ \pm n]$ that are transposed by $\rho_{j}$ belong to the same orbit of $\sigma$.

(2) If $\sigma \leq \tau \leq \omega$ with respect to the partial order coming from $\left(W_{n}, R_{n}\right)$, then every orbit of $\sigma$ is contained in an orbit of $\tau$ (and as a consequence, every fixed point of $\tau$ must also be a fixed point of $\sigma)$.

(3) Let $\tau$ be in $[\varepsilon, \omega], \tau \neq \varepsilon$, and let $\tau=\tau_{1} \cdots \tau_{k}$ be the cycle factorization of type $B$ for $\tau$. If $\sigma_{1} \in\left[\varepsilon, \tau_{1}\right], \ldots, \sigma_{k} \in\left[\varepsilon, \tau_{k}\right]$, then the permutations $\sigma_{1}, \ldots, \sigma_{k}$ commute with each other, and $\sigma:=\sigma_{1} \cdots \sigma_{k}$ is in $[\varepsilon, \tau]$.

(4) Let $\tau$ be in $[\varepsilon, \omega], \tau \neq \varepsilon$, and let $\tau=\tau_{1} \cdots \tau_{k}$ be the cycle factorization of type $B$ for $\tau$. Then every permutation $\sigma \in[\varepsilon, \tau]$ can be uniquely written in the form $\sigma=\sigma_{1} \cdots \sigma_{k}$ where $\sigma_{1} \in\left[\varepsilon, \tau_{1}\right], \ldots, \sigma_{k} \in\left[\varepsilon, \tau_{k}\right]$.

Just as was the case for Theorem 3.2, the above proposition follows easily from its counterpart of type A (Proposition 3.1), via the use of Lemma 3.2. We will also use the following simple observation:

Lemma. Let $Y_{1}, \ldots, Y_{k}$ be non-empty and pairwise disjoint subsets of $[ \pm n]$, such that $Y_{j}=-Y_{j}, 1 \leq j \leq k$. Let $\sigma_{1}, \ldots, \sigma_{k} \in W_{n}$ be such that $\sigma_{j}$ fixes all the elements of $[ \pm n] \backslash Y_{j}, 1 \leq j \leq k$. Set $\sigma:=\sigma_{1} \cdots \sigma_{k}$ (commuting product). Then $|\sigma|=\left|\sigma_{1}\right|+\cdots+\left|\sigma_{k}\right|$.

Proof of the Lemma. This is immediate from the explicit formula for $|\cdot|$ provided by equation (2.9) of Section 2.6.

Proof of the Proposition. (2) Follows by combining Lemma 3.2 with part (2) of Proposition 3.1.

(1) Follows from part (2) of the proposition, and the fact that for every $1 \leq j \leq k$ we have $\rho_{j} \leq \sigma$. (The latter inequality is easily proved directly from the definitions, by showing separately that $\rho_{j} \leq \rho_{1} \cdots \rho_{j}$ and that $\rho_{1} \cdots \rho_{j} \leq \sigma$.)

(3) For every $1 \leq j \leq k$, let us denote by $Y_{j}=-Y_{j}$ the subset of $[ \pm n]$ where $\tau_{j}$ acts non-trivially. From part (2) of the proposition and the hypothesis that $\sigma_{j} \leq \tau_{j}$ it follows that $\sigma_{j}$ fixes all the elements of $[ \pm n] \backslash Y_{j}, 1 \leq j \leq k$. Hence, the preceding lemma can be applied to $\sigma_{1}, \ldots, \sigma_{k}$, and gives us that:

$$
|\sigma|=\left|\sigma_{1}\right|+\cdots+\left|\sigma_{k}\right| \text {. }
$$

Since it is also clear that $\tau_{j}$ and $\sigma_{j}^{-1} \tau_{j}$ fix all the elements of $[ \pm n] \backslash Y_{j}, 1 \leq j \leq k$, the same lemma also gives that:

$$
|\tau|=\left|\tau_{1}\right|+\cdots+\left|\tau_{k}\right|
$$

and

$$
\left|\sigma^{-1} \tau\right|=\left|\sigma_{1}^{-1} \tau_{1}\right|+\cdots+\left|\sigma_{k}^{-1} \tau_{k}\right| .
$$


Now, the fact that $\sigma_{j} \in\left[\varepsilon, \tau_{j}\right]$ is equivalent to $\left|\sigma_{j}\right|+\left|\sigma_{j}^{-1} \tau_{j}\right|=\left|\tau_{j}\right|, 1 \leq j \leq k$. By adding together these equalities for $1 \leq j \leq k$, and by taking (3.5), (3.6), (3.7) into account, we obtain that $|\sigma|+\left|\sigma^{-1} \tau\right|=|\tau|$, i.e., that $\sigma \in[\varepsilon, \tau]$.

(4) Let $Y_{1}, \ldots, Y_{k} \subset[ \pm n]$ have the same significance as in the proof of part (3) (for every $1 \leq j \leq k, \tau_{j}$ acts like $\tau$ on $Y_{j}$ and like $\varepsilon$ on $[ \pm n] \backslash Y_{j}$ ). Since every orbit of $\sigma$ is contained in an orbit of $\tau$ (by part (2) of the proposition), it is immediate that $\sigma$ can be uniquely factored as $\sigma=\sigma_{1} \cdots \sigma_{k}$, where $\sigma_{j}$ fixes the elements of $[ \pm n] \backslash Y_{j}, 1 \leq j \leq k$. Then the preceding lemma applies, and gives us equalities stated exactly as in (3.5), (3.6), (3.7) from the proof of part (3). But then we have

$$
\begin{aligned}
|\tau| & \left.=|\sigma|+\left|\sigma^{-1} \tau\right| \quad \text { (because } \sigma \in[\varepsilon, \tau]\right) \\
& =\sum_{j=1}^{k}\left|\sigma_{j}\right|+\sum_{j=1}^{k}\left|\sigma_{j}^{-1} \tau_{j}\right| \quad(\text { by }(3.5),(3.7)) \\
& =\sum_{j=1}^{k}\left(\left|\sigma_{j}\right|+\left|\sigma_{j}^{-1} \tau_{j}\right|\right) \\
& \geq \sum_{j=1}^{k}\left|\tau_{j}\right| \quad(\text { by the triangle inequality for }|\cdot|) \\
& =|\tau| \quad(\text { by }(3.6)) ;
\end{aligned}
$$

this can happen only if each of the inequalities $\left|\sigma_{j}\right|+\left|\sigma_{j}^{-1} \tau_{j}\right| \geq\left|\tau_{j}\right|$ holds with equality, i.e., if $\sigma_{j} \in\left[\varepsilon, \tau_{j}\right]$ for every $1 \leq j \leq k$.

Remark. Suppose now that $\tau \in[\varepsilon, \omega]$, and $\tau \neq \varepsilon$. Let $\tau=\tau_{1} \cdots \tau_{k}$ be the cycle factorization of type $\mathrm{B}$ for $\tau$, and for every $1 \leq j \leq k$ let $Y_{j}=-Y_{j}$ denote the subset of $[ \pm n]$ where $\tau_{j}$ acts in a non-trivial way. From parts (3) and (4) of the preceding proposition, we get a canonical poset isomorphism:

$$
[\varepsilon, \tau] \cong\left[\varepsilon, \tau_{1}\right] \times \cdots \times\left[\varepsilon, \tau_{k}\right] .
$$

On the other hand, for every $1 \leq j \leq k$ it is immediate (by using part (2) of the preceding proposition) that $\left[\varepsilon, \tau_{j}\right]$ can be identified with an interval going from the identity to a "long cycle" (either inversion-invariant or not inversion-invariant) in the hyperoctahedral group with symbols from $Y_{j}$. By the results of Section 3.2, the latter interval is in turn identified canonically with either $N C^{(B)}\left(\operatorname{card}\left(Y_{j}\right) / 2\right)$ or $N C^{(A)}\left(\operatorname{card}\left(Y_{j}\right) / 2\right)$ (depending on whether $Y_{j}$ was an orbit of $\tau$, or the union of two disjoint orbits of $\tau$, inverse to each other in $[ \pm n])$.

The conclusion of this discussion is the following: If the given $\tau$ has an inversioninvariant orbit $Z=-Z$, and if the other orbits with more than one element for $\tau$ are denoted as $X_{1},-X_{1}, \ldots, X_{p},-X_{p}$, then

$$
[\varepsilon, \tau] \cong N C^{(A)}\left(\operatorname{card}\left(X_{1}\right)\right) \times \cdots \times N C^{(A)}\left(\operatorname{card}\left(X_{p}\right)\right) \times N C^{(B)}(\operatorname{card}(Z) / 2) .
$$

If we are in the opposite case (when $\tau$ has no inversion-invariant orbits), then we denote the orbits with more than one element for $\tau$ as $X_{1},-X_{1}, \ldots, X_{k},-X_{k}$, and we just get

$$
[\varepsilon, \tau] \cong N C^{(A)}\left(\operatorname{card}\left(X_{1}\right)\right) \times \cdots \times N C^{(A)}\left(\operatorname{card}\left(X_{k}\right)\right)
$$

Equations (3.9) and (3.10) represent the type B analogue of equation (3.3) of Section 3.1 . 


\section{REVIEW OF BASIC DEFINITIONS \\ AND FACTS FROM COMBINATORIAL FREE PROBABILITY (OF TYPE A)}

In this section we give a brief glossary of basic definitions and facts which are used in the combinatorics of free probability, and for which "type B analogues" will be proposed in the following sections.

4.1. Non-commutative probability space. The simplest concept of a "noncommutative probability space", focusing only on algebraic and combinatorial aspects, consists of a pair $(\mathcal{A}, \varphi)$, where $\mathcal{A}$ is a complex unital algebra ("the algebra of random variables"), and where $\varphi: \mathcal{A} \rightarrow \mathbf{C}$ ("the expectation") is a linear functional, normalized by the condition that $\varphi(1)=1$.

4.2. Free independence. This concept is defined for a family of unital subalgebras $\mathcal{A}_{1}, \ldots, \mathcal{A}_{k} \subset \mathcal{A}$, where $(\mathcal{A}, \varphi)$ is a non-commutative probability space. The precise definition consists of a "condition in moments": $\mathcal{A}_{1}, \ldots, \mathcal{A}_{k}$ are freely independent if and only if

$$
\left\{\begin{array}{c}
\varphi\left(a_{1} a_{2} \cdots a_{n}\right)=0 \\
\text { whenever } a_{1} \in \mathcal{A}_{i_{1}}, \ldots, a_{n} \in \mathcal{A}_{i_{n}} \\
\text { with } i_{1} \neq i_{2}, i_{2} \neq i_{3}, \ldots, i_{n-1} \neq i_{n} \\
\text { and where } \varphi\left(a_{1}\right)=\cdots=\varphi\left(a_{n}\right)=0
\end{array}\right.
$$

(see e.g. Chapter 2 of [20]).

4.3. Non-crossing cumulants. The non-crossing cumulant functionals associated to a non-commutative probability space $(\mathcal{A}, \varphi)$ were introduced by R. Speicher [17]. They are a family of multilinear functionals

$$
\left(\kappa_{n}^{(A)}: \mathcal{A}^{n} \rightarrow \mathbf{C}\right)_{n=1}^{\infty}
$$

(where the superscript $A$ in " $\kappa_{n}^{(A)}$ " is a reminder that we are dealing with objects "of type A"). The equation which defines the functionals $\kappa_{n}^{(A)}$ is

$$
\sum_{p \in N C^{(A)}(n)} \prod_{F \text { a block of } p} \kappa_{\operatorname{card}(F)}^{(A)}\left(\left(a_{1}, \ldots, a_{n}\right) \mid F\right)=\varphi\left(a_{1} \cdots a_{n}\right),
$$

holding for every $n \geq 1$ and for every $a_{1}, \ldots, a_{n} \in \mathcal{A}$. In (4.2) we used the convention of notation that if $F=\left\{j_{1}<j_{2}<\cdots<j_{m}\right\}$ is a subset of $\{1, \ldots, n\}$ and if $a_{1}, \ldots, a_{n} \in \mathcal{A}$, then

$$
\left(a_{1}, \ldots, a_{n}\right) \mid F:=\left(a_{j_{1}}, a_{j_{2}}, \ldots, a_{j_{m}}\right) \in \mathcal{A}^{m} .
$$

A recursive use of (4.2) gives explicit formulas for the functionals $\kappa_{n}^{(A)}$. For instance, for $n=1,2,3$ we get

$$
\left\{\begin{array}{lll}
\kappa_{1}^{(A)}\left(a_{1}\right) & =\varphi\left(a_{1}\right) \\
\kappa_{2}^{(A)}\left(a_{1}, a_{2}\right) & =\varphi\left(a_{1} a_{2}\right)-\varphi\left(a_{1}\right) \varphi\left(a_{2}\right) \\
\kappa_{3}^{(A)}\left(a_{1}, a_{2}, a_{3}\right)= & \varphi\left(a_{1} a_{2} a_{3}\right) \\
& -\varphi\left(a_{1}\right) \varphi\left(a_{2} a_{3}\right)-\varphi\left(a_{2}\right) \varphi\left(a_{1} a_{3}\right) \\
& -\varphi\left(a_{1} a_{2}\right) \varphi\left(a_{3}\right)+2 \varphi\left(a_{1}\right) \varphi\left(a_{2}\right) \varphi\left(a_{3}\right) .
\end{array}\right.
$$

The main reason for which non-crossing cumulants are an efficient tool in free probability is that they provide a neat reformulation for the definition of free independence. More precisely, we have 
Proposition. Let $(\mathcal{A}, \varphi)$ be a non-commutative probability space, and let $\mathcal{A}_{1}, \ldots$, $\mathcal{A}_{k}$ be unital subalgebras of $\mathcal{A}$. Then $\mathcal{A}_{1}, \ldots, \mathcal{A}_{k}$ are freely independent if and only if:

$$
\left\{\begin{array}{c}
\kappa_{n}^{(A)}\left(a_{1}, a_{2}, \ldots, a_{n}\right)=0 \\
\text { whenever } a_{1} \in \mathcal{A}_{i_{1}}, \ldots, a_{n} \in \mathcal{A}_{i_{n}} \\
\text { and } \exists 1 \leq s<t \leq n \text { such that } i_{s} \neq i_{t} .
\end{array}\right.
$$

The condition in (4.5) is called "vanishing of mixed cumulants". The equivalence between free independence and the vanishing of mixed cumulants was first proved in 17]; an instructive alternative proof of this equivalence appears in Section 6 of 14.

4.4. Moment series and R-transform. Let $(\mathcal{A}, \varphi)$ be a non-commutative probability space, and let $a$ be an element of $\mathcal{A}$. The power series

$$
M_{a}(z):=\sum_{n=1}^{\infty} \varphi\left(a^{n}\right) z^{n}
$$

is called the moment series of $a$ in $(\mathcal{A}, \varphi)$, while the series

$$
R_{a}(z):=\sum_{n=1}^{\infty} \kappa_{n}^{(A)}(a, \ldots, a) z^{n}
$$

is called the R-transform of $a$ in $(\mathcal{A}, \varphi)$. The concept of R-transform was first studied by a method using Toeplitz matrices, in [19], where the following basic fact was proved: if $a, b \in \mathcal{A}$ and if the subalgebra of $\mathcal{A}$ generated by $a$ is freely independent from the one generated by $b$, then

$$
R_{a+b}(z)=R_{a}(z)+R_{b}(z) .
$$

For the developments in the present paper it turns out to be more important to look at the counterpart of (4.8) which expresses the R-transform of the product $a b$ in terms of the series $R_{a}$ and $R_{b}$ (under the same hypothesis that the subalgebras generated by $a$ and $b$ are freely independent). This multiplicative counterpart of (4.8) was analyzed in 13] (in fact, in the more general situation when one deals with $k$-tuples $\left(a_{1}, \ldots, a_{k}\right)$ and $\left(b_{1}, \ldots, b_{k}\right)$ instead of just $a$ and $\left.b\right)$ by introducing a certain operation of "boxed convolution", which is the object of our next section.

\section{Boxed Convolution of type A And of type B}

\subsection{Review of boxed convolution of type A.}

\section{Definition.}

(1) We denote by $\Theta^{(A)}$ the set of power series of the form

$$
f(z)=\sum_{n=1}^{\infty} \alpha_{n} z^{n}
$$

where the $\alpha_{n}$ 's are complex numbers.

(2) On $\Theta^{(A)}$ we define a binary operation $\Theta^{(A)}$, as follows. If

$$
f(z)=\sum_{n=1}^{\infty} \alpha_{n} z^{n} \text { and } g(z)=\sum_{n=1}^{\infty} \beta_{n} z^{n},
$$


then $f \otimes^{(A)} g$ is the series $\sum_{n=1}^{\infty} \gamma_{n} z^{n}$, where

$$
\gamma_{n}:=\sum_{\substack{p \in N C^{(A)}(n) \\ p:=\left\{F_{1}, \ldots, F_{k}\right\} \\ K r(p):=\left\{E_{1}, \ldots, E_{h}\right\}}}\left(\prod_{j=1}^{k} \alpha_{\operatorname{card}\left(F_{j}\right)}\right) \cdot\left(\prod_{i=1}^{h} \beta_{\operatorname{card}\left(E_{i}\right)}\right), n \geq 1 .
$$

The operation $\mathrm{⿴囗}^{(A)}$ was introduced in [13], in the more general situation when we consider (instead of series as in (5.1)) series in $k$ non-commuting indeterminants $z_{1}, \ldots, z_{k}$. For the sake of simplicity, we will limit the considerations of the present paper to the situation when $k=1$. The main point of $[13]$ is that $\mathrm{⿴囗十}^{(A)}$ provides the combinatorial description for the multiplication of two freely independent elements, in terms of their R-transforms. More precisely, we have

Theorem (see [13], Theorem 1.4). Let $(\mathcal{A}, \varphi)$ be a non-commutative probability space, and let $a, b \in \mathcal{A}$ be such that the unital subalgebras of $\mathcal{A}$ generated by a and by $b$ are freely independent. Then the R-transform of the product ab satisfies the equation

$$
R_{a b}=R_{a} \otimes^{(A)} R_{b}
$$

The operation $\mathrm{A}^{(A)}$ is associative, and has the identity series $\Delta(z)=z$ as unit element. This can be checked either directly from the combinatorial definition in (5.2) (as was done in [13]), or by exploiting the interpretation of $\star^{(A)}$ provided by (5.3).

What we want to emphasize in our review here is that $\Theta^{(A)}$ provides the middle ground between free probability (on the one hand) and the Cayley graph framework of Section 2 (on the other hand). The relation between $\otimes^{(A)}$ and free probability is illustrated by the preceding theorem. In order to present the relation between $\star^{(A)}$ and the Cayley graph framework, we first record the remark that $\star^{(A)}$ can be truncated to an operation on $\mathbf{C}^{n}$, for every $n \geq 1$.

Remark. Let $f(z)=\sum_{n=1}^{\infty} \alpha_{n} z^{n}$ and $g(z)=\sum_{n=1}^{\infty} \beta_{n} z^{n}$ be two series in $\Theta^{(A)}$. For every $n \geq 1$, the coefficient $\gamma_{n}$ of order $n$ in the boxed convolution $f$ 太 $^{(A)} g$ is defined by $(5.2)$ as a polynomial expression in $\alpha_{1}, \ldots, \alpha_{n}, \beta_{1}, \ldots, \beta_{n}$. For instance, for $n=1,2,3$ one gets

$$
\left\{\begin{array}{l}
\gamma_{1}=\alpha_{1} \beta_{1}, \\
\gamma_{2}=\alpha_{2} \beta_{1}^{2}+\alpha_{1}^{2} \beta_{2}, \\
\gamma_{3}=\alpha_{3} \beta_{1}^{3}+3 \alpha_{1} \alpha_{2} \beta_{1} \beta_{2}+\alpha_{1}^{3} \beta_{3} .
\end{array}\right.
$$

As a consequence, for a fixed value of $n \geq 1$, it makes sense to define an operation $\otimes_{n}^{(A)}$ on $\mathbf{C}^{n}$, which records how the $n$-tuple of coefficients of order up to $n$ in $f \otimes^{(A)} g$ is obtained from the corresponding $n$-tuples of coefficients in $f$ and in $g$. For example, the equations (5.4) provide the explicit description for the fact that

$$
\left(\gamma_{1}, \gamma_{2}, \gamma_{3}\right)=\left(\alpha_{1}, \alpha_{2}, \alpha_{3}\right) \otimes_{3}^{(A)}\left(\beta_{1}, \beta_{2}, \beta_{3}\right) .
$$

From the properties of $\mathrm{⿴}^{(A)}$ it follows that $\mathrm{⿴囗十}_{n}^{(A)}$ is associative and has unit equal to $(1,0, \ldots, 0) \in \mathbf{C}^{n}$, for every $n \geq 1$. 
But now, the truncated operation $\mathrm{A}_{n}^{(A)}$ turns out to be closely related to the operation of restricted convolution in the Cayley graph framework for the marked group $\left(S_{n}, T_{n}\right)$ discussed in Section 2.5. This fact was observed in [2], and is stated precisely as follows.

Proposition (see 2, Section 3.1). Let $n$ be a fixed positive integer. Consider the marked group $\left(S_{n}, T_{n}\right)$, as discussed in Section 2.5. For every $n$-tuple $\alpha=$ $\left(\alpha_{1}, \ldots, \alpha_{n}\right) \in \mathbf{C}^{n}$ we denote by $u_{\alpha}: S_{n} \rightarrow \mathbf{C}$ the function defined by the following formula:

$$
u_{\alpha}(t)=\alpha_{1}^{k_{1}(t)} \alpha_{2}^{k_{2}(t)} \cdots \alpha_{n}^{k_{n}(t)}, \quad t \in S_{n},
$$

where $k_{m}(t)$ stands for the number of orbits of cardinality $m$ of the permutation $t$ $\left(1 \leq m \leq n, t \in S_{n}\right)$.

Consider, on the other hand, the operation of restricted convolution $*_{r}$ for complex-valued functions on $S_{n}$ (as discussed in Section 2.7). Then the set of functions $\left\{u_{\alpha}: \alpha \in \mathbf{C}^{n}\right\}$ defined by (5.5) is closed under $*_{r}$, and the operation $*_{r}$ on this set of functions coincides with the truncated boxed convolution $\mathrm{⿴}_{n}^{(A)}$. In other words, for every $\alpha, \beta \in \mathbf{C}^{n}$ we have that $u_{\alpha} *_{r} u_{\beta}=u_{\gamma}$, where $\gamma=\alpha \Theta_{n}^{(A)} \beta$.

When put together, the preceding proposition and theorem give a connection between free probability and the Cayley graph framework, which is obtained by using the boxed convolution $\star^{(A)}$ as an intermediate object.

We conclude this subsection by recording another fact about $\mathrm{A}^{(A)}$ which will be useful in the sequel, namely that one can effortlessly define "vector-valued versions" of this operation.

Remark and Notation. Let $\mathcal{C}$ be a unital commutative algebra over $\mathbf{C}$. The formula (5.2) used in the definition of the operation $\boldsymbol{\Xi}^{(A)}$ makes perfect sense if the "scalars" $\alpha_{1}, \alpha_{2}, \alpha_{3}, \ldots$ and $\beta_{1}, \beta_{2}, \beta_{3}, \ldots$ appearing there are elements of $\mathcal{C}$ (the sums and products in $(5.2)$ become sums and products in $\mathcal{C}$ ). When using scalars from $\mathcal{C}$, one obtains a version of the boxed convolution of type $\mathrm{A}$ which will be denoted as $\mathbb{A}_{\mathcal{C}}^{(A)}$.

5.2. Boxed convolution of type B. We will now define the type B analogue $\Theta^{(B)}$, of the operation $\Theta^{(A)}$ discussed in Section 5.1. In order to do so, we will repeat the considerations which related $\otimes^{(A)}$ to the Cayley graph framework, in a context where we replace the symmetric groups by hyperoctahedral groups. (The boxed convolution of type B will eventually become the middle ground between free probability of type B and the Cayley graph framework; but this is not an issue for the moment, since we have not introduced the necessary elements of free probability of type B.)

We start by looking at the type B analogues for the functions $u_{\alpha}$ which appeared in equation (5.5) of Proposition 5.1. By taking into consideration the specifics of the factorization into cycles in type B (as discussed in Notation 3.3) we come to the following:

Notation. Let $n$ be a fixed positive integer, and consider the hyperoctahedral group $W_{n}$. Let $\alpha=\left(\left(\alpha_{1}^{\prime}, \alpha_{1}^{\prime \prime}\right), \ldots,\left(\alpha_{n}^{\prime}, \alpha_{n}^{\prime \prime}\right)\right)$ be an $n$-tuple in $\left(\mathbf{C}^{2}\right)^{n}$. We denote by $u_{\alpha}$ : 
$W_{n} \rightarrow \mathbf{C}$ the function defined by the formula

$$
u_{\alpha}(\tau)=\left(\alpha_{1}^{\prime}\right)^{k_{1}(\tau)}\left(\alpha_{1}^{\prime \prime}\right)^{l_{1}(\tau)} \cdots\left(\alpha_{n}^{\prime}\right)^{k_{n}(\tau)}\left(\alpha_{n}^{\prime \prime}\right)^{l_{n}(\tau)}, \quad \tau \in W_{n}
$$

where, for every $\tau \in W_{n}$ and every $1 \leq m \leq n$, the number $k_{m}(\tau)$ counts the pairs of orbits of $\tau$ that are not inversion-invariant and have cardinality $m$, while $l_{m}(\tau)$ counts the orbits of $\tau$ that are inversion-invariant and have cardinality $2 m$.

Remark. The next thing to do is to examine the restricted convolution of functions $u_{\alpha}$ of the kind introduced before. It is unfortunate that a restricted convolution $u_{\alpha} *_{r} u_{\beta}$, with $\alpha, \beta \in\left(\mathbf{C}^{2}\right)^{n}$, will not generally be a function $u_{\gamma}$ for some $\gamma \in\left(\mathbf{C}^{2}\right)^{n}$. This can be seen by direct computation, already when $n=2$. Indeed, in the case when $n=2$, let us denote the unit of $W_{2}$ by $\varepsilon$, and let us denote

$$
\begin{gathered}
\tau_{1}=\left(\begin{array}{rrrr}
1 & 2 & -1 & -2 \\
-1 & 2 & 1 & -2
\end{array}\right), \tau_{2}=\left(\begin{array}{rrrr}
1 & 2 & -1 & -2 \\
1 & -2 & -1 & 2
\end{array}\right), \\
\tau=\tau_{1} \tau_{2}=\left(\begin{array}{rrrr}
1 & 2 & -1 & -2 \\
-1 & -2 & 1 & 2
\end{array}\right) .
\end{gathered}
$$

Then it is easily seen that every function $u_{\alpha}$ on $W_{2}$ must satisfy the relation

$$
u_{\alpha}(\varepsilon) u_{\alpha}(\tau)=u_{\alpha}\left(\tau_{1}\right) u_{\alpha}\left(\tau_{2}\right)
$$

while a convolution $u_{\alpha} *_{r} u_{\beta}$ does not satisfy this relation (unless some special conditions are imposed on $\alpha$ and $\beta$ ). While concretely verifying the latter fact, the reader will observe that the source of the problem lies in the fact that the permutation $\tau$ in (5.7) can be factored not only as $\tau=\tau_{1} \tau_{2}$, but also as

$$
\tau=\left(\begin{array}{rrrr}
1 & 2 & -1 & -2 \\
2 & 1 & -2 & -1
\end{array}\right) \cdot\left(\begin{array}{rrrr}
1 & 2 & -1 & -2 \\
-2 & -1 & 2 & 1
\end{array}\right)
$$

(where both the factorizations in (5.7) and (5.8) use permutations from the set of generators $R_{2} \subset W_{2}$ considered in Section 2.6). Furthermore, what makes the alternative factorizations in (5.7) and (5.8) coexist is the fact that the permutation $\tau$ that is factored has more than one inversion-invariant orbit.

Now, let us consider again the inversion-invariant long cycle $\omega$, which played a prominent role in Sections 3.2 and 3.3. We know that any permutation $\tau \in[\varepsilon, \omega]$ can have at most one inversion-invariant orbit (as a consequence of Corollary 2.6); so the pathology described previously cannot occur for such $\tau$. In fact, the following is true:

Proposition. Let $n$ be a positive integer, and let $\alpha=\left(\left(\alpha_{1}^{\prime}, \alpha_{1}^{\prime \prime}\right), \ldots,\left(\alpha_{n}^{\prime}, \alpha_{n}^{\prime \prime}\right)\right)$ and $\beta=\left(\left(\beta_{1}^{\prime}, \beta_{1}^{\prime \prime}\right), \ldots,\left(\beta_{n}^{\prime}, \beta_{n}^{\prime \prime}\right)\right)$ be in $\left(\mathbf{C}^{2}\right)^{n}$. Define the numbers $\gamma_{1}^{\prime}, \gamma_{1}^{\prime \prime}, \ldots, \gamma_{n}^{\prime}, \gamma_{n}^{\prime \prime} \in \mathbf{C}$ 
by the formulas

$$
\gamma_{m}^{\prime}=\sum_{\substack{p \in N C^{(A)}(m) \\ p:=\left\{F_{1}, \ldots, F_{k}\right\} \\ K r(p):=\left\{E_{1}, \ldots, E_{h}\right\}}}\left(\prod_{j=1}^{k} \alpha_{\operatorname{card}\left(F_{j}\right)}^{\prime}\right) \cdot\left(\prod_{i=1}^{h} \beta_{\operatorname{card}\left(E_{i}\right)}^{\prime}\right)
$$

$$
\begin{aligned}
& \gamma_{m}^{\prime \prime}=\sum_{\substack{\pi \in N C^{(B)}(m) \text { with zero-block } \\
\pi:=\left\{Z, X_{1},-X_{1}, \ldots, X_{k},-X_{k}\right\} \\
K r(\pi):=\left\{Y_{1},-Y_{1}, \ldots, Y_{h},-Y_{h}\right\}}}\left(\prod_{j=1}^{k} \alpha_{\operatorname{card}\left(X_{j}\right)}^{\prime}\right) \cdot \alpha_{\operatorname{card}(Z) / 2}^{\prime \prime} \cdot\left(\prod_{i=1}^{h} \beta_{\operatorname{card}\left(Y_{i}\right)}^{\prime}\right) \\
& +\sum_{\substack{\pi \in N C^{(B)}(m) \text { without zero-block } \\
\pi:=\left\{X_{1},-X_{1}, \ldots, X_{k},-X_{k}\right\} \\
K r(\pi):=\left\{Z, Y_{1},-Y_{1}, \ldots, Y_{h},-Y_{h}\right\}}}\left(\prod_{j=1}^{k} \alpha_{\operatorname{card}\left(X_{j}\right)}^{\prime}\right) \cdot \beta_{\operatorname{card}(Z) / 2}^{\prime \prime} \cdot\left(\prod_{i=1}^{h} \beta_{\operatorname{card}\left(Y_{i}\right)}^{\prime}\right),
\end{aligned}
$$

for $1 \leq m \leq n$ (and where in (5.10) $Z$ stands for the zero-block of either $\pi$ or $\operatorname{Kr}(\pi))$. Then

$$
\left(u_{\alpha} *_{r} u_{\beta}\right)\left|[\varepsilon, \omega]=u_{\gamma}\right|[\varepsilon, \omega],
$$

where $\gamma:=\left(\left(\gamma_{1}^{\prime}, \gamma_{1}^{\prime \prime}\right), \ldots,\left(\gamma_{n}^{\prime}, \gamma_{n}^{\prime \prime}\right)\right) \in\left(\mathbf{C}^{2}\right)^{n}$.

In the proof of the proposition we will use the following fact.

Lemma. Suppose that $Y_{1}, \ldots, Y_{k}$ are non-empty inversion-invariant subsets of $[ \pm n]$ such that $Y_{i} \cap Y_{j}=\emptyset$ for $i \neq j$, and suppose that $\sigma_{1}, \ldots, \sigma_{k} \in W_{n}$ are such that $\sigma_{j}$ fixes all the elements of $[ \pm n] \backslash Y_{j}, 1 \leq j \leq k$. Let $\alpha$ be in $\left(\mathbf{C}^{2}\right)^{n}$, and consider the function $u_{\alpha}: W_{n} \rightarrow \mathbf{C}$ defined as previously (cf. (5.6)). Then

$$
u_{\alpha}\left(\sigma_{1}\right) \cdots u_{\alpha}\left(\sigma_{k}\right)=u_{\alpha}\left(\sigma_{1} \cdots \sigma_{k}\right) \cdot u_{\alpha}(\varepsilon)^{k-1},
$$

where $\varepsilon$ is the unit of $W_{n}$.

The proof of the lemma is a straightforward application of equation (5.6) defining $u_{\alpha}$, and is left to the reader.

Proof of the Proposition. Note that in the case $m=1$, equation (5.9) gives us that $\gamma_{1}^{\prime}=\alpha_{1}^{\prime} \cdot \beta_{1}^{\prime}$. This shows in particular that $\left(u_{\alpha} *_{r} u_{\beta}\right)(\varepsilon)=u_{\gamma}(\varepsilon)$, since

$$
\left(u_{\alpha} *_{r} u_{\beta}\right)(\varepsilon)=u_{\alpha}(\varepsilon) \cdot u_{\beta}(\varepsilon)=\left(\alpha_{1}^{\prime}\right)^{n} \cdot\left(\beta_{1}^{\prime}\right)^{n},
$$

while $u_{\gamma}(\varepsilon)=\left(\gamma_{1}^{\prime}\right)^{n}$.

For the rest of the proof we fix a permutation $\tau \in[\varepsilon, \omega], \tau \neq \varepsilon$, for which we will prove that $\left(u_{\alpha} *_{r} u_{\beta}\right)(\tau)=u_{\gamma}(\tau)$.

It is immediate that the value of $\left(u_{\alpha} *_{r} u_{\beta}\right)(\tau)$ varies continuously as a function of $(\alpha, \beta) \in\left(\mathbf{C}^{2}\right)^{n} \times\left(\mathbf{C}^{2}\right)^{n}$, and also that $u_{\gamma}(\tau)$ depends continuously on $(\alpha, \beta)$ (since $\gamma$ itself does, as is clear from (5.9) and (5.10)). By making if necessary a small perturbation of $\alpha_{1}^{\prime}$ and $\beta_{1}^{\prime}$ we can, therefore, assume, without loss of generality, that $\alpha_{1}^{\prime} \neq 0 \neq \beta_{1}^{\prime}$. Note that this implies $u_{\alpha}(\varepsilon)=\alpha_{1}^{\prime n} \neq 0, u_{\beta}(\varepsilon)=\beta_{1}^{\prime n} \neq 0$.

The permutation $\tau$ that we fixed has at most one inversion-invariant orbit. Thus there are two cases to consider: when $\tau$ has no inversion-invariant orbit, and when $\tau$ has exactly one inversion-invariant orbit $Z$. We will discuss the latter situation 
(the case without inversion-invariant orbits is analogous; one just has to ignore the part corresponding to $Z$ throughout the computations).

So, let $Z, X_{1},-X_{1}, \ldots, X_{k},-X_{k}$ be the list of orbits of $\tau$ that have more than one element. Let $\tau=\tau_{0} \tau_{1} \cdots \tau_{k}$ be the cycle decomposition of type B for $\tau$ (as discussed in Notation 3.3), where $\tau_{0}$ acts non-trivially on $Z$ and $\tau_{j}$ acts non-trivially on $X_{j} \cup\left(-X_{j}\right), 1 \leq j \leq k$. With this notation it is clear that

$$
u_{\gamma}(\tau)=\gamma_{\text {card }(Z) / 2}^{\prime \prime} \cdot \prod_{j=1}^{k} \gamma_{\text {card }\left(X_{j}\right)}^{\prime} \cdot\left(\gamma_{1}^{\prime}\right)^{n-\operatorname{card}(Z) / 2-\sum_{j=1}^{k} \operatorname{card}\left(X_{j}\right)},
$$

and what we have to prove is that $\left(u_{\alpha} *_{r} u_{\beta}\right)(\tau)$ is also equal to the same quantity.

We compute

$$
\begin{gathered}
\left.\left(u_{\alpha} *_{r} u_{\beta}\right)(\tau)=\sum_{\substack{\sigma \in[\varepsilon, \tau] \\
\sigma_{0} \in\left[\varepsilon, \tau_{0}\right], \ldots \\
\ldots, \sigma_{k} \in\left[\varepsilon, \tau_{k}\right]}} u_{\alpha}(\sigma) u_{\beta}\left(\sigma^{-1} \tau\right) \text { (by the definition of } *_{r}\right) \\
=\sum_{\substack{\sigma_{0} \in\left[\varepsilon, \tau_{0}\right], \ldots \\
\ldots, \sigma_{k} \in\left[\varepsilon, \tau_{k}\right]}}\left(\prod_{j=0}^{k} u_{\alpha}\left(\sigma_{j}\right)\right) \cdot u_{\alpha}\left(\sigma_{0}^{-1} \tau_{0} \cdots \sigma_{k}^{-1} \tau_{k}\right) \text { (by Prop. 3.3) }\left(\prod_{j=0}^{k} u_{\beta}\left(\sigma_{j}^{-1} \tau_{j}\right)\right) \cdot u_{\beta}(\varepsilon)^{-k}
\end{gathered}
$$

(by the lemma preceding this proposition)

$$
=\left(u_{\alpha}(\varepsilon) u_{\beta}(\varepsilon)\right)^{-k} \cdot \prod_{j=0}^{k}\left(\sum_{\sigma_{j} \in\left[\varepsilon, \tau_{j}\right]} u_{\alpha}\left(\sigma_{j}\right) u_{\beta}\left(\sigma_{j}^{-1} \tau_{j}\right)\right) .
$$

At this moment we pick a value of $j \in\{0,1, \ldots, k\}$ and we look at the sum over $\left[\varepsilon, \tau_{j}\right]$ which appeared in (5.14). Exactly as explained at the end of Section 3.3, this sum is converted into a sum over $N C^{(B)}(\operatorname{card}(Z) / 2)$, if $j=0$, and into a sum over $N C^{(A)}\left(\operatorname{card}\left(X_{j}\right)\right)$, if $1 \leq j \leq k$. It is immediately verified that the change of variable from " $\sum_{\sigma_{0} \in\left[\varepsilon, \tau_{0}\right]}$ " to " $\sum_{\pi \in N C(B)}(\operatorname{card}(Z) / 2)$ " takes us to a sum as described in (5.10), thus leading to:

$$
\sum_{\sigma_{0} \in\left[\varepsilon, \tau_{0}\right]} u_{\alpha}\left(\sigma_{0}\right) u_{\beta}\left(\sigma_{0}^{-1} \tau_{0}\right)=\left(\alpha_{1}^{\prime} \beta_{1}^{\prime}\right)^{n-\operatorname{card}(Z) / 2} \cdot \gamma_{\operatorname{card}(Z) / 2}^{\prime \prime} .
$$

(The power of $\alpha_{1}^{\prime} \beta_{1}^{\prime}$ appearing on the right-hand side of (5.15) comes from the $n-\operatorname{card}(Z) / 2$ pairs of fixed points that both $\sigma_{0}$ and $\sigma_{0}^{-1} \tau_{0}$ have in $[ \pm n] \backslash Z$.) Similarly, for $1 \leq j \leq k$ we get

$$
\sum_{\sigma_{j} \in\left[\varepsilon, \tau_{j}\right]} u_{\alpha}\left(\sigma_{j}\right) u_{\beta}\left(\sigma_{j}^{-1} \tau_{j}\right)=\left(\alpha_{1}^{\prime} \beta_{1}^{\prime}\right)^{n-\operatorname{card}\left(X_{j}\right)} \cdot \gamma_{\operatorname{card}\left(X_{j}\right)}^{\prime} .
$$

By replacing (5.15) and (5.16) in (5.14) we come to

$$
\begin{aligned}
& \left(u_{\alpha} *_{r} u_{\beta}\right)(\tau)=\left(u_{\alpha}(\varepsilon) u_{\beta}(\varepsilon)\right)^{-k} \\
& \times\left(\alpha_{1}^{\prime} \beta_{1}^{\prime}\right)^{n(k+1)-\operatorname{card}(Z) / 2-\sum_{j=1}^{k} \operatorname{card}\left(X_{j}\right)} \cdot \gamma_{\operatorname{card}(Z) / 2}^{\prime \prime} \cdot \prod_{j=1}^{k} \gamma_{\operatorname{card}\left(X_{j}\right)}^{\prime} .
\end{aligned}
$$

This is indeed equal to the expression from equation (5.13), since $\left(u_{\alpha}(\varepsilon) u_{\beta}(\varepsilon)\right)^{-k}=\left(\alpha_{1}^{\prime} \beta_{1}^{\prime}\right)^{-n k}$ and $\alpha_{1}^{\prime} \beta_{1}^{\prime}=\gamma_{1}^{\prime}$. 
We are thus led to introduce the type B analogue for the operation of boxed convolution, in the following way.

\section{Definition.}

(1) We denote by $\Theta^{(B)}$ the set of power series of the form

$$
f(z)=\sum_{n=1}^{\infty}\left(\alpha_{n}^{\prime}, \alpha_{n}^{\prime \prime}\right) z^{n}
$$

where the $\alpha_{n}^{\prime}$ 's and $\alpha_{n}^{\prime \prime}$ 's are complex numbers.

(2) Let $f(z):=\sum_{n=1}^{\infty}\left(\alpha_{n}^{\prime}, \alpha_{n}^{\prime \prime}\right) z^{n}$ and $g(z):=\sum_{n=1}^{\infty}\left(\beta_{n}^{\prime}, \beta_{n}^{\prime \prime}\right) z^{n}$ be in $\Theta^{(B)}$. For every $m \geq 1$, consider the numbers $\gamma_{m}^{\prime}$ and $\gamma_{m}^{\prime \prime}$ defined as in (5.9) and (5.10) (in terms of $\alpha_{1}^{\prime}, \alpha_{1}^{\prime \prime}, \ldots, \alpha_{m}^{\prime}, \alpha_{m}^{\prime \prime}$ and $\beta_{1}^{\prime}, \beta_{1}^{\prime \prime}, \ldots, \beta_{m}^{\prime}, \beta_{m}^{\prime \prime}$ ). Then the series $\sum_{n=1}^{\infty}\left(\gamma_{n}^{\prime}, \gamma_{n}^{\prime \prime}\right) z^{n}$ is called the boxed convolution of type B of $f$ and $g$, and is denoted $f$ 淿 $^{(B)} g$.

The proposition proved above has the consequence that

Corollary. The binary operation $\mathrm{⿴}^{(B)}$ defined on $\Theta^{(B)}$ is associative and has the series $\Delta^{\prime}(z)=(1,0) z$ as a unit.

Proof. It is immediate that for every $n \geq 1$ it makes sense to consider the truncation of $\boldsymbol{\star}^{(B)}$ to order $n$, thus obtaining a binary operation $\boldsymbol{⿴ 囗 十}_{n}^{(B)}$ on $\left(\mathbf{C}^{2}\right)^{n}$. (This is analogous to the considerations done for $\Theta^{(A)}$ in Section 5.1.) It clearly suffices to prove that, for every $n \geq 1$, the operation $\Theta_{n}^{(B)}$ is associative and has the element $((1,0),(0,0), \ldots,(0,0)) \in\left(\mathbf{C}^{2}\right)^{n}$ as a unit. But the definition of $\mathbf{⿴ 囗 十}^{(B)}$ is made so that we have

$$
u_{\alpha \otimes_{n}^{(B)} \beta}\left|[\varepsilon, \omega]=\left(u_{\alpha} *_{r} u_{\beta}\right)\right|[\varepsilon, \omega],
$$

for every $n \geq 1$ and every $\alpha, \beta \in\left(\mathbf{C}^{2}\right)^{n}$. Hence, the desired properties of $\boldsymbol{⿴ 囗 十}_{n}^{(B)}$ follow from the corresponding ones for the restricted convolution $*_{r}$ (cf. Proposition $2.7)$.

5.3. A key connection between boxed convolutions of types A and B. In this section we present a way of relating the operations $\mathrm{X}^{(A)}$ and $\mathrm{X}^{(B)}$, which will be crucial for understanding how to move towards free probabilistic considerations of type B. In brief, we will show that $\mathrm{t}^{(B)}$ is still "a $\mathrm{\star}^{(A)}$ operation," but with the scalars replaced by a certain algebra structure on $\mathbf{C}^{2}$.

Definition. Let $\mathcal{C}$ denote $\mathbf{C}^{2}$ with the multiplication given by

$$
\left(\alpha^{\prime}, \alpha^{\prime \prime}\right) \cdot\left(\beta^{\prime}, \beta^{\prime \prime}\right):=\left(\alpha^{\prime} \beta^{\prime}, \alpha^{\prime} \beta^{\prime \prime}+\alpha^{\prime \prime} \beta^{\prime}\right) .
$$

Then $\mathcal{C}$ is a $\mathbf{C}$-algebra, with unit $(1,0)$. This algebra may also be identified with the algebra of 2-by-2 upper triangular Toeplitz matrices $\left[\begin{array}{cc}\alpha^{\prime} & \alpha^{\prime \prime} \\ 0 & \alpha^{\prime}\end{array}\right]$, or with $\mathbf{C}[x] /\left(x^{2}\right)$. This algebra structure on $\mathbf{C}^{2}$ is implicitly present in the considerations of [16], Section 6, page 217.

Recall now that the boxed convolution of type A has a version $\otimes_{\mathcal{C}}^{(A)}$ which is "with coefficients in $\mathcal{C}$ " (cf. the Remark and Notation at the end of Section 5.1). As a set, the space of power series $\Theta_{\mathcal{C}}^{(A)}$ on which we consider the operation $\otimes_{\mathcal{C}}^{(A)}$ 
coincides with the set of power series $\Theta^{(B)}$ of Definition 5.2. In other words, on $\Theta^{(B)}$ there are two operations that we can look at: $\otimes^{(B)}$ and $\otimes_{\mathcal{C}}^{(A)}$.

Theorem. In the notation of the preceding paragraph, $\otimes^{(B)}=\otimes_{\mathcal{C}}^{(A)}$.

Proof. Let $f(z)=\sum_{n=1}^{\infty}\left(\alpha_{n}^{\prime}, \alpha_{n}^{\prime \prime}\right) z^{n}, g(z)=\sum_{n=1}^{\infty}\left(\beta_{n}^{\prime}, \beta_{n}^{\prime \prime}\right) z^{n}$ be in $\Theta^{(B)}$. Write

$$
f \otimes^{(B)} g=: \sum_{n=1}^{\infty}\left(\gamma_{n}^{\prime}, \gamma_{n}^{\prime \prime}\right) z^{n} \text { and } f \Theta_{\mathcal{C}}^{(A)} g=: \sum_{n=1}^{\infty}\left(\delta_{n}^{\prime}, \delta_{n}^{\prime \prime}\right) z^{n}
$$

We fix a positive integer $n$, for which we will show that $\left(\gamma_{n}^{\prime}, \gamma_{n}^{\prime \prime}\right)=\left(\delta_{n}^{\prime}, \delta_{n}^{\prime \prime}\right)$. It will be convenient to use the following notation. First, for $p \in N C^{(A)}(n)$ we will denote (as in Section 1.1) the number of blocks of $p$ by blno $(p)$. Then, given a partition $p \in N C^{(A)}(n)$, we will denote the blocks of $p$ (listed in increasing order of their minimal elements, say) by $F(p, i), 1 \leq i \leq \operatorname{blno}(p)$. It will moreover be convenient that, for every $p \in N C^{(A)}(n)$, we use the notation

$$
F(p, i), \quad \operatorname{blno}(p)<i \leq n+1,
$$

for the blocks of the Kreweras complement $\operatorname{Kr}(p)$. (In other words, we set $F(p, i):=$ $F(\operatorname{Kr}(p), i-\operatorname{blno}(p))$ for $\operatorname{blno}(p)<i \leq n+1$. The indexing up to $n+1$ is correct by virtue of (1.2) in Section 1.1.) Finally, it will also be convenient to set a unified notation for the $\alpha$ 's and $\beta$ 's that appear as coefficients for the series $f$ and $g$ : for every $p \in N C^{(A)}(n)$ and $1 \leq i \leq n+1$ we put

$$
\theta^{\prime}(p, i):= \begin{cases}\alpha^{\prime}{ }^{\operatorname{card}(F(p, i))} & \text { if } i \leq \operatorname{blno}(p), \\ \beta^{\prime}{ } \operatorname{card}(F(p, i)) & \text { if } i>\operatorname{blno}(p),\end{cases}
$$

and

$$
\theta^{\prime \prime}(p, i):= \begin{cases}\alpha^{\prime \prime} \operatorname{card}(F(p, i)) & \text { if } i \leq \operatorname{blno}(p) \\ \beta^{\prime \prime}{ }^{c} \operatorname{card}(F(p, i)) & \text { if } i>\operatorname{blno}(p)\end{cases}
$$

Let us look at $\gamma_{n}^{\prime}$ and $\gamma_{n}^{\prime \prime}$. First we have

$$
\begin{aligned}
\gamma_{n}^{\prime} & =\sum_{\substack{p \in N C^{(A)}(n) \\
p:=\left\{F_{1}, \ldots, F_{k}\right\} \\
\operatorname{Kr}(p):=\left\{E_{1}, \ldots, E_{h}\right\}}}\left(\prod_{j=1}^{k}\left(\alpha^{\prime} \operatorname{card}\left(E_{j}\right)\right)\right) \cdot\left(\prod_{i=1}^{h} \beta^{\prime} \operatorname{card}\left(F_{i}\right)\right) \\
& =\sum_{p \in N C^{(A)}(n)} \prod_{i=1}^{n+1} \theta^{\prime}(p, i) .
\end{aligned}
$$

With $\gamma_{n}^{\prime \prime}$ the situation would seem to be more complicated, because this coefficient is defined via a summation over $N C^{(B)}(n)$, as described by equation (5.10) (where one replaces $m$ by $n$ ). However, the summation over $N C^{(B)}(n)$ can be reduced to one over $N C^{(A)}(n)$, by using the $(n+1)$-to-1 cover Abs : $N C^{(B)}(n) \rightarrow N C^{(A)}(n)$ which is discussed in Section 1.4. When doing so, and when taking into account the explicit description of $\mathrm{Abs}^{-1}(p)\left(p \in N C^{(A)}(n)\right)$ provided by the proof of Theorem 1.4 , one gets

$$
\gamma_{n}^{\prime \prime}=\sum_{p \in N C^{(A)}(n)}\left(\sum_{m=1}^{n+1} \theta^{\prime \prime}(p, m) \cdot \prod_{i \neq m} \theta^{\prime}(p, i)\right) .
$$


On the other hand, by recalling the definition of the operation $\otimes_{\mathcal{C}}^{(A)}$, we see that $\left(\delta^{\prime}{ }_{n}, \delta^{\prime \prime}{ }_{n}\right)$ equals

$$
\begin{gathered}
\sum_{\substack{p \in N C^{(A)}(n) \\
p:=\left\{F_{1}, \ldots, F_{k}\right\} \\
\operatorname{Kr}(p):=\left\{E_{1}, \ldots, E_{h}\right\}}}\left(\prod_{j=1}^{k}\left(\alpha^{\prime}{ }_{\operatorname{card}\left(E_{j}\right)}, \alpha^{\prime \prime} \operatorname{card}\left(E_{j}\right)\right)\right) \cdot\left(\prod_{i=1}^{h}\left(\beta^{\prime}{ }_{\operatorname{card}\left(F_{i}\right)}, \beta^{\prime \prime}{ }_{\operatorname{card}\left(F_{i}\right)}\right)\right) \\
\quad=\sum_{p \in N C^{(A)}(n)} \prod_{i=1}^{n+1}\left(\theta^{\prime}(p, i), \theta^{\prime \prime}(p, i)\right)
\end{gathered}
$$

(products considered with respect to the multiplication on the algebra $\mathcal{C}$ )

$$
=\sum_{p \in N C^{(A)}(n)}\left(\prod_{i=1}^{n+1} \theta^{\prime}(p, i), \sum_{m=1}^{n+1} \theta^{\prime \prime}(p, m) \cdot \prod_{i \neq m} \theta^{\prime}(p, i)\right) .
$$

By comparing (5.22) against $(5.20)+(5.21)$, we obtain $\left(\gamma_{n}^{\prime}, \gamma_{n}^{\prime \prime}\right)=\left(\delta_{n}^{\prime}, \delta_{n}^{\prime \prime}\right)$, as desired.

\section{Non-CRossing CUmulants of tyPe B}

In order to define the type B analogue for non-crossing cumulants, we will pursue the idea of using the same equation as for non-crossing cumulants of type A ((4.2) in Section 4.3), but make it have coefficients in $\mathbf{C}^{2}$, where $\mathbf{C}^{2}$ has the algebra structure from Definition 5.3. We have to begin the discussion by introducing the appropriate framework of non-commutative probability space.

\subsection{Non-commutative probability space of type B.}

Definition. By a non-commutative probability space of type $B$ we will understand a system $(\mathcal{A}, \varphi, \mathcal{V}, f, \Phi)$ where:

(1) $\mathcal{A}$ and $\varphi$ form a non-commutative probability space of type $\mathrm{A}$.

(2) $\mathcal{V}$ is a vector space over $\mathbf{C}$, and $f: \mathcal{V} \rightarrow \mathbf{C}$ is a linear functional.

(3) $\Phi: \mathcal{A} \times \mathcal{V} \times \mathcal{A} \rightarrow \mathcal{V}$ is a two-sided action of $\mathcal{A}$ on $\mathcal{V}$. Usually we will simply write " $a \xi b$ " instead of $\Phi(a, \xi, b)$, for $a, b \in \mathcal{A}$ and $\xi \in \mathcal{V}$.

Remark. Let $(\mathcal{A}, \varphi, \mathcal{V}, f, \Phi)$ be a non-commutative probability space of type B. On the vector space $\mathcal{A} \times \mathcal{V}$ we have a structure of unital algebra (sometimes called "the linking algebra of the bimodule $\mathcal{V}$ "), with multiplication defined by

$$
(a, \xi) \cdot(b, \eta):=(a b, a \eta+\xi b), \quad a, b \in \mathcal{A}, \xi, \eta \in \mathcal{V} .
$$

In other words, the algebra structure on $\mathcal{A} \times \mathcal{V}$ is the one obtained when $(a, \xi) \in$ $\mathcal{A} \times \mathcal{V}$ is identified with a $2 \times 2$ matrix,

$$
(a, \xi) \leftrightarrow\left[\begin{array}{cc}
a & \xi \\
0 & a
\end{array}\right] .
$$

The unit of $\mathcal{A} \times \mathcal{V}$ is $(I, 0)$, where $I$ is the unit of $\mathcal{A}$.

Moreover, we have a natural linear map $E: \mathcal{A} \times \mathcal{V} \rightarrow \mathbf{C}^{2}$, defined by

$$
E((a, \xi)):=(\varphi(a), f(\xi)), \quad a \in \mathcal{A}, \xi \in \mathcal{V} .
$$




\subsection{Non-crossing cumulant functionals of type B.}

Definition. Let $(\mathcal{A}, \varphi, \mathcal{V}, f, \Phi)$ be a non-commutative probability space of type B. The non-crossing cumulant functionals for this space are a family of multilinear functionals $\left(\kappa_{n}^{(B)}:(\mathcal{A} \times \mathcal{V})^{n} \rightarrow \mathbf{C}^{2}\right)_{n=1}^{\infty}$, uniquely determined by the following equation: for every $n \geq 1$ and every $a_{1}, \ldots, a_{n} \in \mathcal{A}, \xi_{1}, \ldots, \xi_{n} \in \mathcal{V}$ we have that: (6.3)

$$
\sum_{p \in N C^{(A)}(n)} \prod_{\substack{F \text { block } \\ \text { of } p}} \kappa_{\text {card }(F)}^{(B)}\left(\left(a_{1}, \xi_{1}\right), \ldots,\left(a_{n}, \xi_{n}\right) \mid F\right)=E\left(\left(a_{1}, \xi_{1}\right) \cdots\left(a_{n}, \xi_{n}\right)\right) .
$$

In (6.3) the following conventions of notation were used.

- On the left-hand side of (6.3), if $F=\left\{j_{1}<j_{2}<\cdots<j_{m}\right\}$ is a subset of $\{1, \ldots, n\}$, then $\left(\left(a_{1}, \xi_{1}\right), \ldots,\left(a_{n}, \xi_{n}\right) \mid F\right)$ stands for $\left(\left(a_{j_{1}}, \xi_{j_{1}}\right), \ldots,\left(a_{j_{m}}, \xi_{j_{m}}\right)\right)$ $\in(\mathcal{A} \times \mathcal{V})^{m}$. The product indexed by " $F$ block of $p$ " is considered with respect to the multiplication on $\mathbf{C}^{2}$ defined in Section 5.3.

- On the right-hand side of (6.3), $E: \mathcal{A} \times \mathcal{V} \rightarrow \mathbf{C}^{2}$ is as defined in (6.2), and the product $\left(a_{1}, \xi_{1}\right) \cdots\left(a_{n}, \xi_{n}\right) \in \mathcal{A} \times \mathcal{V}$ is computed according to the multiplication rule of (6.1). It is actually straightforward to write the result of this multiplication explicitly:

$$
\left(a_{1}, \xi_{1}\right) \cdots\left(a_{n}, \xi_{n}\right)=\left(a_{1} \cdots a_{n}, \sum_{m=1}^{n} a_{1} \cdots a_{m-1} \xi_{m} a_{m+1} \cdots a_{n}\right) .
$$

Remarks. 1. Equation (6.3) defining the functionals $\kappa_{n}^{(B)}$ has on its left-hand side a summation over $N C^{(A)}(n)$. However, when we concentrate on the second component of this equation, we will really encounter a summation over $N C^{(B)}(n)$, because of exactly the same phenomenon which led to the formula $\star^{(B)}=\star_{\mathcal{C}}^{(A)}$ of Theorem 5.3. This point will be reappearing in Section 6.5 below - cf. the remark at the end of that section.

2. As in type $\mathrm{A}$, the recursive use of equation (6.3) gives explicit formulas for the functionals $\kappa_{n}^{(B)}$. These formulas repeat those describing the functionals $\kappa_{n}^{(A)}$ (as discussed in Section 4.3), with the difference that now we operate in $\mathbf{C}^{2}$ instead of C. For instance,

$$
\kappa_{1}^{(B)}((a, \xi))=E((a, \xi))=(\varphi(a), f(\xi)),
$$

while for $\kappa_{2}^{(B)}$ we get

$$
\begin{gathered}
\kappa_{2}^{(B)}\left(\left(a_{1}, \xi_{1}\right),\left(a_{2}, \xi_{2}\right)\right)=E\left(\left(a_{1}, \xi_{1}\right)\left(a_{2}, \xi_{2}\right)\right)-E\left(\left(a_{1}, \xi_{1}\right)\right) E\left(\left(a_{2}, \xi_{2}\right)\right) \\
=\left(\varphi\left(a_{1} a_{2}\right), f\left(a_{1} \xi_{2}\right)+f\left(\xi_{1} a_{2}\right)\right)-\left(\varphi\left(a_{1}\right) \varphi\left(a_{2}\right), \varphi\left(a_{1}\right) f\left(\xi_{2}\right)+f\left(\xi_{1}\right) \varphi\left(a_{2}\right)\right) \\
=\left(\varphi\left(a_{1} a_{2}\right)-\varphi\left(a_{1}\right) \varphi\left(a_{2}\right), \quad \begin{array}{c}
f\left(a_{1} \xi_{2}\right)-\varphi\left(a_{1}\right) f\left(\xi_{2}\right) \\
\left.+f\left(\xi_{1} a_{2}\right)\right)-f\left(\xi_{1}\right) \varphi\left(a_{2}\right)
\end{array}\right) .
\end{gathered}
$$

3. The equations defining the functionals $\kappa_{n}^{(B)}$ are close to those used in the framework of operator-valued cumulants developed in [18, where $\mathbf{C}^{2}$ plays the role of the algebra of scalars. There are, however, some details that are different here, namely that

(a) $\mathbf{C}^{2}$ is not canonically embedded inside $\mathcal{A} \times \mathcal{V}$, and

(b) the map $E$ is not required to be a conditional expectation (actually $\mathcal{A} \times \mathcal{V}$ does not even carry a canonical structure of bimodule over $\mathbf{C}^{2}$ ). 
4. Considering (6.6), it is natural to ask whether one could not also describe the functionals $\kappa_{n}^{(B)}$ by specifying each component. The first component of $\kappa_{2}^{(B)}\left(\left(a_{1}, \xi_{1}\right),\left(a_{2}, \xi_{2}\right)\right)$ is just $\kappa_{2}^{(A)}\left(a_{1}, a_{2}\right)$. In order to describe what happens on the second component, we will have to discuss yet another variation of the cumulant functional of type A, where one of the arguments is allowed to be a vector. It is worth mentioning that exactly this variation of the cumulant functional has recently appeared in [15], in connection with the study of Voiculescu's concept of free Fisher information.

6.3. The functionals $\kappa_{n ; m}^{\left(A^{\prime}\right)}$. Let us observe that equation (4.2) defining the functional $\kappa_{n}^{(A)}$ still makes sense when one of the arguments $a_{1}, \ldots, a_{n}$ of the functional is allowed to be a vector in an $\mathcal{A}$-bimodule (rather than just being an element of $\mathcal{A})$. We formalize this observation as follows.

Definition. Let $(\mathcal{A}, \varphi, \mathcal{V}, f, \Phi)$ be a non-commutative probability space of type B. For every $n \geq 1$ and every $m \in\{1, \ldots, n\}$ we denote by $\kappa_{n ; m}^{\left(A^{\prime}\right)}$ the multilinear functional from $\mathcal{A}^{m-1} \times \mathcal{V} \times \mathcal{A}^{n-m}$ to $\mathbf{C}$ which is defined by exactly the same formula as for $\kappa_{n}^{(A)}: \mathcal{A}^{n} \rightarrow \mathbf{C}$, but where the $m$ th argument is a vector from $\mathcal{V}$, and where " $\varphi$ " is replaced by " $f$ " in all the appropriate places.

Referring to the explicit low-order formulas presented in equations (4.4) of Section 4.3 , we have for instance that $\kappa_{2 ; 1}^{\left(A^{\prime}\right)}: \mathcal{V} \times \mathcal{A} \rightarrow \mathbf{C}$ and $\kappa_{2 ; 2}^{\left(A^{\prime}\right)}: \mathcal{A} \times \mathcal{V} \rightarrow \mathbf{C}$ are defined by

$$
\left\{\begin{array}{l}
\kappa_{2 ; 1}^{\left(A^{\prime}\right)}(\xi, a)=f(\xi a)-f(\xi) \varphi(a) \\
\kappa_{2 ; 2}^{\left(A^{\prime}\right)}(a, \xi)=f(a \xi)-\varphi(a) f(\xi)
\end{array}\right.
$$

for $\xi \in \mathcal{V}$ and $a \in \mathcal{A}$, or that $\kappa_{3 ; 2}^{\left(A^{\prime}\right)}: \mathcal{A} \times \mathcal{V} \times \mathcal{A} \rightarrow \mathbf{C}$ is given by $\kappa_{3 ; 2}^{\left(A^{\prime}\right)}\left(a, \xi, a^{\prime}\right)=f\left(a \xi a^{\prime}\right)-\varphi(a) f\left(\xi a^{\prime}\right)-f(\xi) \varphi\left(a a^{\prime}\right)-f(a \xi) \varphi\left(a^{\prime}\right)+2 \varphi(a) f(\xi) \varphi\left(a^{\prime}\right)$, for $a, a^{\prime} \in \mathcal{A}$ and $\xi \in \mathcal{V}$. The general equation (analogous to (4.2) from Section 4.3) that determines the functionals $\kappa_{n ; m}^{\left(A^{\prime}\right)}$ can be written as follows. For $n \geq m \geq 1$ and for $a_{1}, \ldots, a_{m-1}, a_{m+1}, \ldots, a_{n} \in \mathcal{A}, \xi \in \mathcal{V}$ we have

$$
\begin{gathered}
=\sum_{p \in N C^{(A)}(n)}^{f\left(a_{1} \cdots a_{m-1} \xi a_{m+1} \cdots a_{n}\right)}\left[\kappa_{\operatorname{card}\left(F_{o}\right) ; j}^{\left(A^{\prime}\right)}\left(a_{1}, \ldots, a_{m-1}, \xi, a_{m+1}, \ldots, a_{n} \mid F_{o}\right)\right. \\
\left.\cdot \prod_{\substack{F \text { block of } p, F \neq F_{o}}} \kappa_{\operatorname{card}(F)}^{(A)}\left(a_{1}, \ldots, a_{m-1}, \xi, a_{m+1}, \ldots, a_{n} \mid F\right)\right],
\end{gathered}
$$

where $F_{o}$ denotes the block of $p$ that contains $m$, and $j$ denotes the position of $m$ inside $F_{o}$ (i.e, if $F_{o}=\left\{i_{1}<i_{2}<\cdots<i_{l}\right\}$, then $i_{j}=m$ ).

Remark (unified notation for $\kappa_{n}^{(A)}$ and $\left.\kappa_{m ; n}^{\left(A^{\prime}\right)}\right)$. Let $(\mathcal{A}, \varphi, \mathcal{V}, f, \Phi)$ be a non-commutative probability space of type $B$. In view of the fact that the cumulant functionals $\kappa_{n}^{(A)}: \mathcal{A}^{n} \rightarrow \mathbf{C}$ and $\kappa_{n ; m}^{\left(A^{\prime}\right)}: \mathcal{A}^{m-1} \times \mathcal{V} \times \mathcal{A}^{n-m} \rightarrow \mathbf{C}$ are governed by exactly the same combinatorics, it is convenient to use a unified notation for these two types of cumulants. More precisely, it is convenient to deal with expressions of the form

$$
\text { " } \kappa_{n}^{\left(A^{\prime}\right)}\left(x_{1}, \ldots, x_{n}\right) "
$$


where

$$
\left\{\begin{array}{l}
\circ \quad \text { either all of } x_{1}, \ldots, x_{n} \text { are in } \mathcal{A}, \\
\quad \text { and } \kappa_{n}^{\left(A^{\prime}\right)}\left(x_{1}, \ldots, x_{n}\right):=\kappa_{n}^{(A)}\left(x_{1}, \ldots, x_{n}\right) \\
\circ \quad \text { or there is a specified } m \in\{1, \ldots, n\} \text { such that } x_{m} \in \mathcal{V}, \\
\quad \text { while } x_{1}, \ldots, x_{m-1}, x_{m+1}, \ldots, x_{n} \in \mathcal{A}, \\
\quad \text { in this case, } \kappa_{n}^{\left(A^{\prime}\right)}\left(x_{1}, \ldots, x_{n}\right):=\kappa_{n ; m}^{\left(A^{\prime}\right)}\left(x_{1}, \ldots, x_{n}\right) .
\end{array}\right.
$$

The use of the notation (6.9) simplifies for instance the above equation (6.8), in the sense that it is no longer necessary to distinguish the block $F_{o}$ of $p$ in the expression on the right-hand side of (6.8). Indeed, the term indexed by $p \in$ $N C^{(A)}(n)$ in the sum appearing there is now simply written as

$$
\prod_{F \text { block of } p} \kappa_{\text {card }(F)}^{\left(A^{\prime}\right)}\left(a_{1}, \ldots, a_{m-1}, \xi, a_{m+1}, \ldots, a_{n} \mid F\right) .
$$

Moreover, following a convention commonly used in the theory of non-crossing cumulants of type A, it is convenient to introduce the following notation.

Notation. Let $(\mathcal{A}, \varphi, \mathcal{V}, f, \Phi)$ be a non-commutative probability space of type B. Let $n$ be a positive integer, and let $p$ be a partition in $N C^{(A)}(n)$. For $x_{1}, \ldots, x_{n}$ as described in (6.10), we denote

$$
\kappa_{p}^{\left(A^{\prime}\right)}\left(x_{1}, \ldots, x_{n}\right):=\prod_{F \text { block of } p} \kappa_{\text {card }(F)}^{\left(A^{\prime}\right)}\left(x_{1}, \ldots, x_{n} \mid F\right) .
$$

In the first situation covered by (6.10) (when $x_{1}, \ldots, x_{n} \in \mathcal{A}$ ), the quantity appearing in (6.12) is precisely what is commonly denoted as $\kappa_{p}^{(A)}\left(x_{1}, \ldots, x_{n}\right)$ in the theory of non-crossing cumulants of type A. In the second situation covered by (6.10) (when $x_{m} \in \mathcal{V}$ for a specified $m \in\{1, \ldots, n\}$ ), the quantity appearing in (6.12) coincides with the one from (6.11).

Let us observe that if we use the above notation, then equation (6.8) defining the cumulants of type $A^{\prime}$ can be written in the more compressed form

$$
f\left(a_{1} \cdots a_{m-1} \xi a_{m+1} \cdots a_{n}\right)=\sum_{p \in N C^{(A)}(n)} \kappa_{p}^{\left(A^{\prime}\right)}\left(a_{1}, \ldots, a_{m-1}, \xi, a_{m+1}, \ldots, a_{n}\right),
$$

for $n \geq m \geq 1$ and $a_{1}, \ldots, a_{m-1}, a_{m+1}, \ldots, a_{n} \in \mathcal{A}, \xi \in \mathcal{V}$.

We will conclude this subsection by recording two facts which are very basic for the theory of cumulants of type A, and which have straightforward generalizations to cumulants of type $A^{\prime}$.

Proposition. Let $(\mathcal{A}, \varphi, \mathcal{V}, f, \Phi)$ be a non-commutative probability space of type $B$. Let $n \geq 2$ be an integer, and consider some $x_{1}, \ldots, x_{n}$ as described in (6.10). Then we have the relation:

$$
\begin{aligned}
& \kappa_{n-1}^{\left(A^{\prime}\right)}\left(x_{1}, \ldots x_{r-1}, x_{r} x_{r+1}, x_{r+2}, \ldots, x_{n}\right)=\kappa_{n}^{\left(A^{\prime}\right)}\left(x_{1}, \ldots, x_{n}\right) \\
& +\sum_{\substack{p \in N C^{(A)}(n) \text { with blno }(p)=2, p \text { separates } r \text { from } r+1}} \kappa_{p}^{\left(A^{\prime}\right)}\left(x_{1}, \ldots, x_{n}\right) .
\end{aligned}
$$

Note that on the left-hand side of (6.14) we may have that $x_{r} x_{r+1} \in \mathcal{A}$ (if $x_{r}, x_{r+1} \in \mathcal{A}$ ), or we may have that $x_{r} x_{r+1} \in \mathcal{V}$ (if one of $x_{r}, x_{r+1}$ is from $\mathcal{V}$ 
while the other one is from $\mathcal{A}$ ). The sum over $p$ on the right-hand side has $n-1$ terms, which could easily be listed explicitly (the possible choices for $p$ are $p=$ $\{(1, \ldots, r),(r+1, \ldots n)\}, p=\{(l, \ldots, r),(1, \ldots, l-1, r+1, \ldots, n)\}$ for some $2 \leq l \leq r$, and $p=\{(1, \ldots, r, l+1, \ldots, n),(r+1, \ldots, l)\}$ for some $r+1 \leq l \leq n-1)$.

The relation (6.14) is copied from the theory of cumulants of type $\mathrm{A}-$ see [17, or the generalizations obtained in 10 . The proof of (6.14) is also copied verbatim from the type A situation (cf. 17], proof of Proposition 1 on page 622); this is due to the fact that the cumulants $\kappa_{n}^{\left(A^{\prime}\right)}$ obey exactly the same combinatorics as the cumulants $\kappa_{n}^{(A)}$.

Corollary. Let $(\mathcal{A}, \varphi, \mathcal{V}, f, \Phi)$ be a non-commutative probability space of type B. Let $n \geq 2$ be an integer, let $m$ be in $\{1, \ldots, n\}$, and consider $\xi \in \mathcal{V}$ and $a_{1}, \ldots, a_{m-1}$, $a_{m+1}, \ldots, a_{n} \in \mathcal{A}$. If there exists an index $r \in\{1, \ldots, n\} \backslash\{m\}$ such that $a_{r} \in \mathbf{C} I$, then

$$
\kappa_{n}^{\left(A^{\prime}\right)}\left(a_{1}, \ldots, a_{m-1}, \xi, a_{m+1}, \ldots, a_{n}\right)=0 .
$$

(Sketch of) Proof. A well-known fact in the theory of cumulants of type A is that

$$
\kappa_{n}^{(A)}\left(a_{1}, \ldots, a_{n}\right)=0
$$

whenever $n \geq 2$ and there exists $r \in\{1, \ldots, n\}$ such that $a_{r} \in \mathbf{C} I$ (see e.g. [17, page 624). The proof of (6.15) is easily obtained by induction on $n$, by using (6.16) and the recurrence relation (6.14). (Group $x_{r}$ with $x_{r+1}$ where $x_{r}=a_{r} \in \mathbf{C} I$. Then note that in the situation at hand, most of the terms on the right-hand side of (6.14) will vanish, either because of the induction hypothesis or because of (6.16).)

6.4. Relation between the functionals $\kappa_{n}^{(B)}$ and $\kappa_{n}^{\left(A^{\prime}\right)}$. We can now give the following alternative description for the non-crossing cumulants of type B:

Theorem. Let $(\mathcal{A}, \varphi, \mathcal{V}, f, \Phi)$ be a non-commutative probability space of type B. Let $n$ be a positive integer, and consider the non-crossing cumulant functional $\kappa_{n}^{(B)}$ : $(\mathcal{A} \times \mathcal{V})^{n} \rightarrow \mathbf{C}^{2}$. Then

$$
\begin{gathered}
\kappa_{n}^{(B)}\left(\left(a_{1}, \xi_{1}\right), \ldots,\left(a_{n}, \xi_{n}\right)\right) \\
=\left(\kappa_{n}^{(A)}\left(a_{1}, \ldots, a_{n}\right), \sum_{m=1}^{n} \kappa_{n}^{\left(A^{\prime}\right)}\left(a_{1}, \ldots, a_{m-1}, \xi_{m}, a_{m+1}, \ldots, a_{n}\right)\right),
\end{gathered}
$$

for every $a_{1}, \ldots, a_{n} \in \mathcal{A}$ and $\xi_{1}, \ldots, \xi_{n} \in \mathcal{V}$.

Proof. For every $n \geq 1$ let us denote by $\lambda_{n}$ the multilinear functional from $(\mathcal{A} \times \mathcal{V})^{n}$ to $\mathbf{C}^{2}$ defined by the right-hand side of (6.17). That is

$$
\begin{gathered}
\lambda_{n}\left(\left(a_{1}, \xi_{1}\right), \ldots,\left(a_{n}, \xi_{n}\right)\right) \\
:=\left(\kappa_{n}^{(A)}\left(a_{1}, \ldots, a_{n}\right), \sum_{m=1}^{n} \kappa_{n}^{\left(A^{\prime}\right)}\left(a_{1}, \ldots a_{m-1}, \xi_{m}, a_{m+1}, \ldots a_{n}\right)\right),
\end{gathered}
$$

for $a_{1}, \ldots, a_{n} \in \mathcal{A}, \xi_{1}, \ldots, \xi_{n} \in \mathcal{V}$. We want to show that $\lambda_{n}=\kappa_{n}^{(B)}, \forall n \geq 1$. In order to do so, it will suffice to verify that equation (6.3), which uniquely determines the functionals $\left(\kappa_{n}^{(B)}\right)_{n=1}^{\infty}$, is also satisfied by the functionals $\left(\lambda_{n}\right)_{n=1}^{\infty}$. In other 
words, it will suffice to show that

$$
\begin{gathered}
\sum_{p \in N C^{(A)}(n)} \prod_{F \text { block of } p} \lambda_{\operatorname{card}(F)}\left(\left(a_{1}, \xi_{1}\right), \ldots,\left(a_{n}, \xi_{n}\right) \mid F\right) \\
=E\left(\left(a_{1}, \xi_{1}\right), \ldots,\left(a_{n}, \xi_{n}\right)\right),
\end{gathered}
$$

for every $n \geq 1$ and every $a_{1}, \ldots, a_{n} \in \mathcal{A}, \xi_{1}, \ldots, \xi_{n} \in \mathcal{V}$. We fix $n \geq 1$ and $a_{1}, \ldots, a_{n} \in \mathcal{A}, \xi_{1}, \ldots, \xi_{n} \in \mathcal{V}$, for which we prove that (6.19) holds.

Let us also fix for the moment a partition $p \in N C^{(A)}(n)$, and let us focus on the term indexed by $p$ in the sum on the left-hand side of (6.19). We compute:

$$
\begin{gathered}
\prod_{F \text { block of } p} \lambda_{\operatorname{card}(F)}\left(\left(a_{1}, \xi_{1}\right), \ldots,\left(a_{n}, \xi_{n}\right) \mid F\right) \\
=\prod_{F \text { block of } p}\left(\kappa^{(A)} \operatorname{card}(F)\left(a_{1}, \ldots, a_{n} \mid F\right),\right. \\
\left.\sum_{m \in F} \kappa_{\operatorname{card}(F)}^{\left(A^{\prime}\right)}\left(a_{1}, \ldots, a_{m-1}, \xi_{m}, a_{m+1}, \ldots, a_{n} \mid F\right)\right)
\end{gathered}
$$

(by substituting the two components of $\lambda_{\operatorname{card}(F)}\left(\left(a_{1}, \xi_{1}\right), \ldots,\left(a_{n}, \xi_{n}\right) \mid F\right)$ from $(6.18)$ )

$$
\begin{aligned}
& =\left(\prod_{\substack{F \text { block } \\
\text { of } p}} \kappa_{\text {card }(F)}^{(A)}\left(a_{1}, \ldots, a_{n} \mid F\right),\right. \\
& \\
& \left.\quad \sum_{m=1}^{n} \kappa_{p}^{\left(A^{\prime}\right)}\left(a_{1}, \ldots, a_{m-1}, \xi_{m}, a_{m+1}, \ldots, a_{n}\right)\right)
\end{aligned}
$$

(by taking into account how the multiplication on $\mathbf{C}^{2}$ was defined in Section 5.3, and by using the notation for $\kappa_{p}^{\left(A^{\prime}\right)}$ from equation (6.12) in Notation 6.3).

If we now sum over $p$ in (6.20), and if we take into account the summation formulas described in (4.2) of Section 4.3 (for the first component) and (6.13) of Section 6.3 (for the second component), then we find that the left-hand side of (6.19) is equal to

$$
\left(\varphi\left(a_{1} \cdots a_{n}\right), \sum_{m=1}^{n} f\left(a_{1} \cdots a_{m-1} \xi_{m} a_{m+1} \cdots a_{n}\right)\right) .
$$

But it is immediately seen that the right-hand side of (6.19) is also equal to (6.21) (cf. (6.4) and (6.2) in Section 6.1).

6.5. Moment series and R-transform of type B. We can now introduce the type $\mathrm{B}$ analogues for the concepts of moment series and R-transform which were reviewed in Section 4.4 .

Definition. Let $(\mathcal{A}, \varphi, \mathcal{V}, f, \Phi)$ be a non-commutative probability space of type $\mathrm{B}$, and consider a couple $(a, \xi) \in \mathcal{A} \times \mathcal{V}$. The moment series and R-transform of $(a, \xi)$ are the power series $M$ and respectively $R$ defined as follows:

$$
M(z):=\sum_{n=1}^{\infty} E\left((a, \xi)^{n}\right) z^{n}
$$




$$
R(z):=\sum_{n=1}^{\infty} \kappa_{n}^{(B)}(\underbrace{(a, \xi), \ldots,(a, \xi)}_{n}) z^{n} .
$$

$M$ and $R$ belong to $\Theta^{(B)}$, the space of power series introduced in Definition 5.2. The expressions " $E\left((a, \xi)^{n}\right)$ " in (6.22) are computed according to the rules described in Remark 6.1.

A basic fact in the theory of the R-transform in type A is that the moment series and the R-transform of a given element are related to each other via a convolution formula (using the operation $\otimes^{(A)}$ ). More precisely, if $(\mathcal{A}, \varphi)$ is a non-commutative probability space of type $\mathrm{A}$, if $a \in \mathcal{A}$, and if $M_{a}, R_{a} \in \Theta^{(A)}$ are the moment series and the R-transform of $a$, then we have

$$
M_{a}=R_{a} \otimes^{(A)} \zeta
$$

where $\zeta(z):=\sum_{n=1}^{\infty} z^{n}$. The analogue of type B for (6.24) is described as follows.

Proposition. Let $(\mathcal{A}, \varphi, \mathcal{V}, f, \Phi)$ be a non-commutative probability space of type $B$, and consider a couple $(a, \xi) \in \mathcal{A} \times \mathcal{V}$. Then the moment series $M$ and the $R$-transform $R$ of $(a, \xi)$ are related by the formula

$$
M=R \otimes^{(B)} \zeta^{\prime},
$$

where $\zeta^{\prime} \in \Theta^{(B)}$ is the series $\sum_{n=1}^{\infty}(1,0) z^{n}$.

Proof. For every $n \geq 1$ we have that the coefficient of order $n$ in $R \otimes^{(B)} \zeta^{\prime}$ is the same as the coefficient of order $n$ in $R \otimes_{\mathcal{C}}^{(A)} \zeta^{\prime}$ (by Theorem 5.3). When we express this coefficient by using the $\mathcal{C}$-valued version of equation (5.2), and when we take into account that all the coefficients of $\zeta^{\prime}$ are equal to the unit of $\mathcal{C}$, we obtain:

$$
\sum_{p \in N C^{(A)}(n)} \prod_{F \text { block of } p}\left(\begin{array}{c}
\text { coefficient of } \\
\text { order card }(F) \text { in } R
\end{array}\right) .
$$

The latter quantity equals

$$
\sum_{p \in N C^{(A)}(n)} \prod_{F \text { block of } p} \kappa_{\text {card }(F)}^{(B)}((a, \xi), \ldots,(a, \xi))
$$

(by the definition of $R$ in (6.23)), and is hence equal to $E\left((a, \xi)^{n}\right)$ by (6.3). But this is precisely the coefficient of order $n$ in $M$.

Remark. Equation (6.25) is equivalent to the particular case of (6.3) from Section 6.2 , where the couples $\left(a_{1}, \xi_{1}\right), \ldots,\left(a_{n}, \xi_{n}\right)$ in $(6.3)$ are all equal to each other. If we had developed the more involved framework which would allow us to define the operation $\star^{(B)}$ for series in $k$ non-commuting indeterminates, then we could now state a more general form of equation (6.25), equivalent to the general case of (6.3).

The essential feature of (6.25) is that on the second component of its right-hand side we encounter a summation over half of the lattice $N C^{(B)}(n)$ - or more precisely, over $\left\{\pi \in N C^{(B)}(n): \pi\right.$ has a zero-block $\}$. (The definition of $\star^{(B)}$ would imply a summation over all of $N C^{(B)}(n)$, but the terms indexed by $\pi$ 's without a zeroblock are killed because of the special form of $\zeta^{\prime}$.) For instance, taking the second component of the coefficient of $z^{2}$ on the two sides of (6.25) yields the formula

$$
f(a \xi+\xi a)=\left[\kappa_{2}^{\left(A^{\prime}\right)}(\xi, a)+\kappa_{2}^{\left(A^{\prime}\right)}(a, \xi)\right]+\left[\kappa_{1}^{\left(A^{\prime}\right)}(\xi) \kappa_{1}^{(A)}(a)\right]+\left[\kappa_{1}^{(A)}(a) \kappa_{1}^{\left(A^{\prime}\right)}(\xi)\right],
$$


where the three groups of terms on the right-hand side are (respectively) the contributions of the partitions $\{(1,2,-1,-2)\},\{(1,-1),(2),(-2)\},\{(1),(-1),(2,-2)\}$ $\in N C^{(B)}(2)$.

\section{Free independence of type B}

Let $(\mathcal{A}, \varphi, \mathcal{V}, f, \Phi)$ be a non-commutative probability space of type B. We will discuss the concept of free independence for a family $\left(\mathcal{A}_{1}, \mathcal{V}_{1}\right), \ldots,\left(\mathcal{A}_{k}, \mathcal{V}_{k}\right)$, where $\mathcal{A}_{1}, \ldots, \mathcal{A}_{k}$ are unital subalgebras of $\mathcal{A}$ and $\mathcal{V}_{1}, \ldots, \mathcal{V}_{k}$ are linear subspaces of $\mathcal{V}$ such that $\mathcal{V}_{j}$ is invariant under the (two-sided) action of $\mathcal{A}_{j}, 1 \leq j \leq k$. Since we have a type $\mathrm{B}$ analogue for non-crossing cumulants, our approach will go via the counterpart of type B for the condition of "vanishing of mixed cumulants" (described in type A by equation (4.5) of Section 4.3).

\subsection{Vanishing mixed cumulants in type B.}

Definition. Let $(\mathcal{A}, \varphi, \mathcal{V}, f, \Phi)$ be a non-commutative probability space of type B. Let $\mathcal{A}_{1}, \ldots, \mathcal{A}_{k}$ be unital subalgebras of $\mathcal{A}$ and let $\mathcal{V}_{1}, \ldots, \mathcal{V}_{k}$ be linear subspaces of $\mathcal{V}$ such that $\mathcal{V}_{j}$ is invariant under the action of $\mathcal{A}_{j}$, for $1 \leq j \leq k$. We say that $\left(\mathcal{A}_{1}, \mathcal{V}_{1}\right), \ldots,\left(\mathcal{A}_{k}, \mathcal{V}_{k}\right)$ have vanishing mixed cumulants of type $B$ if the following condition holds:

$$
\left\{\begin{array}{c}
\kappa_{n}^{(B)}\left(\left(a_{1}, \xi_{1}\right), \ldots,\left(a_{n}, \xi_{n}\right)\right)=0 \\
\text { whenever } a_{1} \in \mathcal{A}_{i_{1}}, \ldots, a_{n} \in \mathcal{A}_{i_{n}}, \xi_{1} \in \mathcal{V}_{i_{1}}, \ldots, \xi_{n} \in \mathcal{V}_{i_{n}} \\
\text { and } \exists 1 \leq s<t \leq n \text { such that } i_{s} \neq i_{t} .
\end{array}\right.
$$

Proposition. Let $(\mathcal{A}, \varphi, \mathcal{V}, f, \Phi)$ and $\mathcal{A}_{1}, \ldots, \mathcal{A}_{k} \subset \mathcal{A}, \mathcal{V}_{1}, \ldots, \mathcal{V}_{k} \subset \mathcal{V}$ be as above, such that $\left(\mathcal{A}_{1}, \mathcal{V}_{1}\right), \ldots,\left(\mathcal{A}_{k}, \mathcal{V}_{k}\right)$ have vanishing cumulants of type $B$. Then the following happen:

1. $\mathcal{A}_{1}, \ldots, \mathcal{A}_{k}$ are freely independent in $(\mathcal{A}, \varphi)$ (in the type $A$ sense).

2. Suppose that $m, n \geq 0$ and that we have $m+n+1$ indices $i_{m}, \ldots, i_{1}, h, j_{1}, \ldots, j_{n}$ in $\{1, \ldots, k\}$ such that any two consecutive indices in this list are different from each other $\left(i_{m} \neq i_{m-1}, \ldots, i_{1} \neq h \neq j_{1}, \ldots, j_{n-1} \neq j_{n}\right)$. Suppose moreover that we have elements $a_{m} \in \mathcal{A}_{i_{m}}, \ldots, a_{1} \in \mathcal{A}_{i_{1}}, \xi \in \mathcal{V}_{h}, b_{1} \in \mathcal{A}_{j_{1}}, \ldots, b_{n} \in \mathcal{A}_{j_{n}}$ such that

$$
\varphi\left(a_{m}\right)=\cdots=\varphi\left(a_{1}\right)=0=\varphi\left(b_{1}\right)=\cdots=\varphi\left(b_{n}\right) \text {. }
$$

Then

$$
f\left(a_{m} \cdots a_{1} \xi b_{1} \cdots b_{n}\right)=0
$$

when $m \neq n$, and

$$
f\left(a_{m} \cdots a_{1} \xi b_{1} \cdots b_{n}\right)=\delta_{i_{1}, j_{1}} \cdots \delta_{i_{n}, j_{n}} \varphi\left(a_{1} b_{1}\right) \cdots \varphi\left(a_{n} b_{n}\right) f(\xi)
$$

when $m=n$.

Proof. 1. If we put $\xi_{1}=\cdots=\xi_{n}=0$ in (7.1), and look at the first component of the equality stated there, then we get precisely equation (4.5) in Proposition 4.3. Hence, the free independence of $\mathcal{A}_{1}, \ldots, \mathcal{A}_{k}$ follows from Proposition 4.3.

2. We expand $f\left(a_{m} \cdots a_{1} \xi b_{1} \cdots b_{n}\right)$ as a sum, by using (6.13) from Section 6.3:

$$
\begin{gathered}
f\left(a_{m} \cdots a_{1} \xi b_{1} \cdots b_{n}\right)=\sum_{p \in N C^{(A)}(m+n+1)} \kappa_{p}^{\left(A^{\prime}\right)}\left(a_{m}, \ldots, a_{1}, \xi, b_{1}, \ldots, b_{n}\right) \\
=\sum_{p \in N C^{(A)}(m+n+1)}\left(\prod_{F \text { block of } p} \kappa_{\text {card }(F)}^{\left(A^{\prime}\right)}\left(a_{m}, \ldots, a_{1}, \xi, b_{1}, \ldots b_{n} \mid F\right)\right) .
\end{gathered}
$$


Let us now pick a non-crossing partition $p \in N C^{(A)}(m+n+1)$. We consider conditions which force the term indexed by $p$ in the sum on the right-hand side of (7.4) to be 0.

Condition 1. $p$ has a singleton block $F=\{s\}$ with $s \neq m+1$.

Indeed, if $s<m+1$, then

$$
\kappa_{\operatorname{card}(F)}^{(A)}\left(a_{m}, \ldots, a_{1}, \xi, b_{1}, \ldots b_{n} \mid F\right)=\kappa_{1}^{(A)}\left(a_{s}\right)=\varphi\left(a_{s}\right)=0,
$$

and the term indexed by $p$ in (7.4) is 0. A similar argument applies if $s>m+1$.

Condition 2. $p$ has a block $F$ that includes two successive numbers.

Indeed, since any two consecutive indices in the list $\left(i_{m}, \ldots, i_{1}, h, j_{1}, \ldots, j_{n}\right)$ are different, the hypothesis of vanishing of mixed cumulants of type B will give us in this case that

$$
\kappa_{m+n+1}^{(B)}\left(\left(a_{1}, 0\right), \ldots,\left(a_{m}, 0\right),(0, \xi),\left(b_{1}, 0\right), \ldots,\left(b_{n}, 0\right) \mid F\right)=(0,0) .
$$

If $F \not \ngtr m+1$, then we project (7.5) on its first component; while if $F \ni m+1$, then we project (7.5) on its second component. In either case, by using Theorem 6.4 , we obtain that

$$
\kappa_{m+n+1}^{\left(A^{\prime}\right)}\left(a_{1}, \ldots, a_{m}, \xi, b_{1}, \ldots, b_{n} \mid F\right)=0 .
$$

This in turn implies that the term indexed by $p$ in (7.4) is equal to 0 .

Condition 3. $p$ has a block containing two distinct elements of $X=\{1, \ldots, m\}$ or of $Y=\{m+2, \ldots, m+n+1\}$.

Indeed, in this case the non-crossing condition implies that $p$ has either a singleton block or a block containing two successive numbers in $X$ or $Y$. Thus $p$ satisfies one of the conditions (1) or (2), and, therefore, the summand indexed by $p$ in (7.4) is zero.

Suppose $p$ is a non-crossing partition such that the term indexed by $p$ in (7.4) is non-zero. Because of the non-crossing condition, $p$ has a block which is a singleton or an interval of length $\geq 2$. Because of the discussion concerning conditions (1) and (2), such a block can only be the singleton $\{m+1\}$. It follows, moreover, from the discussion concerning condition (3) that any other block contains exactly two elements, one from $\{1, \ldots, m\}$ and the other from $\{m+2, \ldots, m+n+1\}$. But this is only possible if $m=n$. Moreover, if $m=n$, the only non-crossing partition with this block structure is $p=\{(1,2 n+1),(2,2 n), \ldots,(n, n+2),(n+1)\}$.

Hence, in the case when $m \neq n$, all the terms of the sum in (7.4) vanish, and we obtain (7.2), while for $m=n$, the sum on the right-hand side of (7.4) reduces to only one term:

$$
f\left(a_{m} \cdots a_{1} \xi b_{1} \cdots b_{n}\right)=\kappa_{2}^{(A)}\left(a_{n}, b_{n}\right) \cdots \kappa_{2}^{(A)}\left(a_{1}, b_{1}\right) \cdot \kappa_{1}^{\left(A^{\prime}\right)}(\xi) .
$$

It is immediate that $\kappa_{2}^{(A)}\left(a_{r}, b_{r}\right)=\delta_{i_{r}, j_{r}} \varphi\left(a_{r} b_{r}\right)$, for every $1 \leq r \leq n$, and it is clear that $\kappa_{1}^{\left(A^{\prime}\right)}(\xi)=f(\xi)$, so that (7.3) follows from (7.6).

7.2. Free independence of type $\mathbf{B}$, in terms of moments. The considerations of Section 7.1 prompt us to make the following definition:

Definition. Let $(\mathcal{A}, \varphi, \mathcal{V}, f, \Phi)$ be a non-commutative probability space of type B. Let $\mathcal{A}_{1}, \ldots, \mathcal{A}_{k}$ be unital subalgebras of $\mathcal{A}$ and let $\mathcal{V}_{1}, \ldots, \mathcal{V}_{k}$ be linear subspaces of $\mathcal{V}$ such that $\mathcal{V}_{j}$ is invariant under the action of $\mathcal{A}_{j}$, for $1 \leq j \leq k$. We will say that $\left(\mathcal{A}_{1}, \mathcal{V}_{1}\right), \ldots,\left(\mathcal{A}_{k}, \mathcal{V}_{k}\right)$ are freely independent if the following happen:

(i) $\mathcal{A}_{1}, \ldots, \mathcal{A}_{k}$ are freely independent in $(\mathcal{A}, \varphi)$ (in the type $\mathrm{A}$ sense). 
(ii) We have the formula

$$
f\left(a_{m} \cdots a_{1} \xi b_{1} \cdots b_{n}\right)= \begin{cases}0, & \text { if } m \neq n, \\ \delta_{i_{1}, j_{1}} \cdots & \delta_{i_{n}, j_{n}} \varphi\left(a_{1} b_{1}\right) \cdots \varphi\left(a_{n} b_{n}\right) f(\xi), \\ & \text { if } m=n,\end{cases}
$$

holding in the following context:

- $m, n$ are non-negative integers;

- $i_{m}, \ldots, i_{1}, h, j_{1}, \ldots, j_{n}$ in $\{1, \ldots, k\}$ are such that any two consecutive indices in the list are different from each other;

- $a_{m} \in \mathcal{A}_{i_{m}}, \ldots, a_{1} \in \mathcal{A}_{i_{1}}, \xi \in \mathcal{V}_{h}, b_{1} \in \mathcal{A}_{j_{1}}, \ldots, b_{n} \in \mathcal{A}_{j_{n}}$ are such that $\varphi\left(a_{m}\right)=\cdots=\varphi\left(a_{1}\right)=0=\varphi\left(b_{1}\right)=\cdots=\varphi\left(b_{n}\right)$.

Remark. Let $(\mathcal{A}, \varphi, \mathcal{V}, f, \Phi)$ be a non-commutative probability space of type $\mathrm{B}$, and let $E: \mathcal{A} \times \mathcal{V} \rightarrow \mathbf{C}^{2}$ be as defined in (6.2). Let $1 \in \mathcal{A}_{1}, \ldots, \mathcal{A}_{k} \subset \mathcal{A}$ be subalgebras, and let $\mathcal{V}_{1}, \ldots, \mathcal{V}_{k} \subset \mathcal{V}$ be linear subspaces such that $\mathcal{V}_{j}$ is invariant under the action of $\mathcal{A}_{j}$, for $1 \leq j \leq k$. We denote

$\mathcal{A}_{o}:=$ the subalgebra generated by $\mathcal{A}_{1} \cup \cdots \cup \mathcal{A}_{k}$, and

$\mathcal{V}_{o}:=$ the smallest linear subspace of $\mathcal{V}$ that contains $\mathcal{V}_{1} \cup \cdots \cup \mathcal{V}_{k}$ and is invariant under the action of $\mathcal{A}_{1}, \ldots, \mathcal{A}_{k}$.

If $\left(\mathcal{A}_{1}, \mathcal{V}_{1}\right), \ldots,\left(\mathcal{A}_{k}, \mathcal{V}_{k}\right)$ are freely independent, then $E \mid \mathcal{A}_{o} \times \mathcal{V}_{o}$ is completely determined by the restrictions $E\left|\mathcal{A}_{1} \times \mathcal{V}_{1}, \ldots, E\right| \mathcal{A}_{k} \times \mathcal{V}_{k}$.

This statement amounts to two things.

(a) That $\varphi \mid \mathcal{A}_{o}$ is completely determined by $\varphi\left|\mathcal{A}_{1}, \ldots, \varphi\right| \mathcal{A}_{k}$. This is a basic consequence of the fact that $\mathcal{A}_{1}, \ldots, \mathcal{A}_{k}$ are freely independent in $(\mathcal{A}, \varphi)$ in the type $\mathrm{A}$ sense - see [20], Section 2.5.

(b) That $f \mid \mathcal{V}_{o}$ is completely determined by $\varphi\left|\mathcal{A}_{1}, \ldots, \varphi\right| \mathcal{A}_{k}$ and by $f\left|\mathcal{V}_{1}, \ldots, f\right| \mathcal{V}_{k}$. The phenomenon here is that $\mathcal{V}_{o}$ is the linear span of vectors of the form

$$
a_{m} \cdots a_{1} \xi b_{1} \cdots b_{n},
$$

where $a_{m}, \ldots, a_{1}, \xi, b_{1}, \ldots, b_{n}$ are as in part (ii) of the preceding definition. (The straightforward verification of the latter fact is left to the reader.)

Proposition. Let $(\mathcal{A}, \varphi, \mathcal{V}, f, \Phi)$ be a non-commutative probability space of type B. Let $\mathcal{A}_{1}, \ldots, \mathcal{A}_{k}$ be unital subalgebras of $\mathcal{A}$ and let $\mathcal{V}_{1}, \ldots, \mathcal{V}_{k}$ be linear subspaces of $\mathcal{V}$ such that $\mathcal{V}_{j}$ is invariant under the action of $\mathcal{A}_{j}$, for $1 \leq j \leq k$. If $\left(\mathcal{A}_{1}, \mathcal{V}_{1}\right), \ldots,\left(\mathcal{A}_{k}, \mathcal{V}_{k}\right)$ are freely independent, then

$$
\left\{\begin{array}{c}
\kappa_{n}^{\left(A^{\prime}\right)}\left(a_{1}, \ldots, a_{m-1}, \xi, a_{m+1}, \ldots, a_{n}\right)=0 \\
\text { whenever } 1 \leq m \leq n, a_{1} \in \mathcal{A}_{i_{1}}, \ldots, a_{m-1} \in \mathcal{A}_{i_{m-1}}, \\
\xi \in \mathcal{V}_{i_{m}}, a_{m+1} \in \mathcal{A}_{i_{m+1}}, \ldots, a_{n} \in \mathcal{A}_{i_{n}} \\
\text { and } \exists 1 \leq s<t \leq n \text { such that } i_{s} \neq i_{t} .
\end{array}\right.
$$

Proof. By induction on $n$. The case $n=1$ is fulfilled vacuously. In the case $n=2$ we have to check that

$$
\kappa_{2}^{\left(A^{\prime}\right)}(\xi, a)=\kappa_{2}^{\left(A^{\prime}\right)}(a, \xi)=0
$$

when $a \in \mathcal{A}_{i}, \xi \in \mathcal{V}_{j}$ and $i \neq j(1 \leq i, j \leq k)$. This follows immediately by using the hypothesis of free independence and the concrete formulas for $\kappa_{2}^{\left(A^{\prime}\right)}(\xi, a)$ and $\kappa_{2}^{\left(A^{\prime}\right)}(a, \xi)$, which were mentioned in Section 6.3. Indeed, for instance, for $\kappa_{2}^{\left(A^{\prime}\right)}(\xi, a)$ 
the hypothesis of free independence gives that $f(\xi(a-\varphi(a) I))=0$, hence that $f(\xi a)=\varphi(a) f(\xi)$, and then

$$
\kappa_{2}^{\left(A^{\prime}\right)}(\xi, a) \stackrel{(6.7)}{=} f(\xi a)-f(\xi) \varphi(a)=0 .
$$

The bulk of the proof will be devoted to the induction step: we fix $n \geq 3$, and we will prove that (7.8) is true for $n$, by assuming that (7.8) was already proved for $1,2, \ldots, n-1$.

Consider some $m \in\{1, \ldots, n\}$ and some $a_{1} \in \mathcal{A}_{i_{1}}, \ldots, a_{m-1} \in \mathcal{A}_{i_{m-1}}, \xi \in$ $\mathcal{V}_{i_{m}}, a_{m+1} \in \mathcal{A}_{i_{m+1}}, \ldots, a_{n} \in \mathcal{A}_{i_{n}}$, where $1 \leq i_{1}, \ldots, i_{n} \leq k$ and there exist $1 \leq s<$ $t \leq n$ such that $i_{s} \neq i_{t}$. We will treat separately the following two cases:

Case 1. There exists $r, 1 \leq r<n$, such that $i_{r}=i_{r+1}$.

Case 1 is treated by using the recurrence formula described in (6.14) of Proposition 6.3 , where we denote $x_{m}=\xi$ and $x_{r}=a_{r}$ for $1 \leq r \leq n, r \neq m$, and where we group $x_{r}$ with $x_{r+1}$ for a value of $r$ such that $i_{r}=i_{r+1}$. Indeed, (6.14) will give us that:

$$
\begin{aligned}
& \kappa_{n}^{\left(A^{\prime}\right)}\left(a_{1}, \ldots, a_{m-1}, \xi, a_{m+1}, \ldots, a_{n}\right)=\kappa_{n}^{\left(A^{\prime}\right)}\left(x_{1}, \ldots, x_{n}\right) \\
& =\kappa_{n-1}^{\left(A^{\prime}\right)}\left(x_{1}, \ldots, x_{r-1}, x_{r} x_{r+1}, x_{r+2}, \ldots x_{n}\right) \\
& -\sum_{\substack{p \in N C^{(A)}(n) \text { with blno }(p)=2 \\
p \text { separates } r \text { from } r+1}} \kappa_{p}^{\left(A^{\prime}\right)}\left(x_{1}, \ldots, x_{n}\right) .
\end{aligned}
$$

Then each of the terms in (7.9) is equal to 0 , either because of the induction hypothesis, or because of a phenomenon of vanishing of mixed cumulants from $\mathcal{A}_{1}, \ldots, \mathcal{A}_{k}$ (in the type A sense).

Case 2. We have $i_{r} \neq i_{r+1}, \forall 1 \leq r<n$.

When treating Case 2, note first that $\kappa_{n}^{\left(A^{\prime}\right)}\left(a_{1}, \ldots, a_{m-1}, \xi, a_{m+1}, \ldots, a_{n}\right)$ does not change its value when we replace $a_{r}$ by $a_{r}-\varphi\left(a_{r}\right) I$, for $1 \leq r \leq n, r \neq m$; indeed, this is an immediate consequence of the multilinearity of $\kappa_{n}^{\left(A^{\prime}\right)}$ combined with Corollary 6.3. By doing these replacements, we can assume without loss of generality that $\varphi\left(a_{r}\right)=0$ for all $r \in\{1, \ldots, n\} \backslash\{m\}$. But then the "word" $a_{1} \cdots a_{m-1} \xi a_{m+1} \cdots a_{n}$ is exactly of the kind considered in equation (7.7) from the definition of free independence, and the hypothesis that $\left(\mathcal{A}_{1}, \mathcal{V}_{1}\right), \ldots,\left(\mathcal{A}_{k}, \mathcal{V}_{k}\right)$ are freely independent gives us

$$
\begin{aligned}
& f\left(a_{1} \cdots a_{m-1} \xi a_{m+1} \cdots a_{n}\right) \\
& \quad= \begin{cases}0, & \text { if } n \neq 2 m-1, \\
\delta_{i_{1}, i_{n}} \cdots & \delta_{i_{m-1}, i_{m+1}} \varphi\left(a_{1} a_{n}\right) \cdots \varphi\left(a_{m-1} a_{m+1}\right) f(\xi), \\
& \text { if } n=2 m-1 .\end{cases}
\end{aligned}
$$

On the other hand, we know from (6.13) that

$$
\left.-\sum_{\substack{p \in N C^{(A)}(n) \\ p \neq 1_{n}}}^{\kappa_{n}^{\left(A^{\prime}\right)}\left(a_{1}, \ldots, a_{m-1}, \xi, a_{m+1}, \ldots, a_{n}\right)=f\left(a_{1} \cdots a_{m-1} \xi a_{m+1} \cdots a_{n}\right)} \prod_{F \text { block of } p} \kappa_{\operatorname{card}(F)}^{\left(A^{\prime}\right)}\left(a_{1}, \ldots, a_{m-1}, \xi, a_{m+1}, \ldots, a_{n} \mid F\right)\right) .
$$

Most of the terms in the sum that was subtracted on the right-hand side of (7.11) are equal to 0 . In fact, one can examine the three conditions listed in the proof of 
Proposition 7.1, and argue that, for $p$ satisfying at least one of the three conditions, the term indexed by $p$ in the sum on the right-hand side of (7.11) vanishes. Namely, if $p$ has a singleton block $F=\{s\}$, where $s \neq m$, then

$$
\kappa_{1}^{(A)}\left(a_{1}, \ldots, a_{m-1}, \xi, a_{m+1}, \ldots, a_{n} \mid F\right)=\kappa_{1}^{(A)}\left(a_{s}\right)=\varphi\left(a_{s}\right)=0 .
$$

In case $p$ has a block $F$ containing two successive numbers $s, s+1$, we have

$$
\kappa_{\operatorname{card}(F)}^{\left(A^{\prime}\right)}\left(a_{1}, \ldots, a_{m-1}, \xi, a_{m+1}, \ldots, a_{n} \mid F\right)=0,
$$

by the induction assumption, since $\operatorname{card}(F)<n$ and $i_{s} \neq i_{s+1}$. Finally, if $p$ has a block $F$ containing two elements of $X=\{1, \ldots, m-1\}$ or of $Y=\{m+1, \ldots, n\}$, then (exactly as in the proof of Proposition 7.1) either $p$ has a singleton block $F^{\prime}=\{s\}$ with $s \neq m$, or $p$ has a block $F^{\prime}$ containing two successive numbers (and one of the two preceding arguments applies).

As observed in the proof of Proposition 7.1, the non-crossing partitions satisfying at least one of the three conditions will cover all non-crossing partitions, if $n \neq 2 m-$ 1 ; and will cover all non-crossing partitions with the exception of $p=\{(1, n),(2, n-$ $1), \ldots,(m-1, m+1),(m)\}$, if $n=2 m-1$. Thus from $(7.11)$ we get

$$
\begin{aligned}
& \kappa_{n}^{\left(A^{\prime}\right)}\left(a_{1}, \ldots, a_{m-1}, \xi, a_{m+1}, \ldots, a_{n}\right) \\
& =\left\{\begin{array}{l}
f\left(a_{1} \cdots a_{m-1} \xi a_{m+1} \cdots a_{n}\right), \text { if } n \neq 2 m-1, \\
f\left(a_{1} \cdots a_{m-1} \xi a_{m+1} \cdots a_{n}\right) \\
-\kappa_{2}^{(A)}\left(a_{1}, a_{n}\right) \cdots \kappa_{2}^{(A)}\left(a_{m-1}, a_{m+1}\right) \kappa_{1}^{\left(A^{\prime}\right)}(\xi), \text { if } n=2 m-1,
\end{array}\right. \\
& =0 \quad \text { (by equation }(7.10)) \text {. }
\end{aligned}
$$

Corollary. Let $(\mathcal{A}, \varphi, \mathcal{V}, f, \Phi)$ be a non-commutative probability space of type $B$. Let $\mathcal{A}_{1}, \ldots, \mathcal{A}_{k}$ be unital subalgebras of $\mathcal{A}$ and let $\mathcal{V}_{1}, \ldots, \mathcal{V}_{k}$ be linear subspaces of $\mathcal{V}$ such that $\mathcal{V}_{j}$ is invariant under the action of $\mathcal{A}_{j}$, for $1 \leq j \leq k$. Then

$\left(\mathcal{A}_{1}, \mathcal{V}_{1}\right), \ldots,\left(\mathcal{A}_{k}, \mathcal{V}_{k}\right)$ are freely independent (in the sense of Definition 7.2) if and only if

$\left(\mathcal{A}_{1}, \mathcal{V}_{1}\right), \ldots,\left(\mathcal{A}_{k}, \mathcal{V}_{k}\right)$ have vanishing mixed cumulants of type $B$ (in the sense of Definition 7.1).

Proof. " $\Leftarrow "$ is the content of Proposition 7.1.

" $\Rightarrow$ " Let $\left(a_{1}, \xi_{1}\right) \in \mathcal{A}_{i_{1}} \times \mathcal{V}_{i_{1}}, \ldots,\left(a_{n}, \xi_{n}\right) \in \mathcal{A}_{i_{n}} \times \mathcal{V}_{i_{n}}$, with $1 \leq i_{1}, \ldots, i_{n} \leq k$, and suppose that there exist $1 \leq s<t \leq n$ such that $i_{s} \neq i_{t}$. We have to show that $\kappa_{n}^{(B)}\left(\left(a_{1}, \xi_{1}\right), \ldots,\left(a_{n}, \xi_{n}\right)\right)=0$. The two components of $\kappa_{n}^{(B)}\left(\left(a_{1}, \xi_{1}\right), \ldots,\left(a_{n}, \xi_{n}\right)\right)$ are $\kappa_{n}^{(A)}\left(a_{1}, \ldots, a_{n}\right)$ and $\sum_{m=1}^{n} \kappa_{n}^{\left(A^{\prime}\right)}\left(a_{1}, \ldots, a_{m-1}, \xi_{m}, a_{m+1}, \ldots, a_{n}\right)$ (cf. Theorem 6.4). The first of these two components vanishes because of the free independence of $\mathcal{A}_{1}, \ldots, \mathcal{A}_{k}$ in $(\mathcal{A}, \varphi)$ (in the type A sense), while the second of the two components vanishes by the preceding proposition.

7.3. R-transforms for sums and products of free elements, in type B. In order to make a case that (similarly to what we had in type A) the operation $\star^{(B)}$ provides the middle ground between the Cayley graph framework and free probability of type B, we now have to prove the type B analogue for Theorem 5.1. We have 
Theorem. Let $(\mathcal{A}, \varphi, \mathcal{V}, f, \Phi)$ be a non-commutative probability space of type B. Let $\mathcal{A}_{1}, \mathcal{A}_{2}$ be unital subalgebras of $\mathcal{A}$, let $\mathcal{V}_{1}, \mathcal{V}_{2}$ be linear subspaces of $\mathcal{V}$ such that $\mathcal{V}_{j}$ is invariant under the action of $\mathcal{A}_{j}(j=1,2)$, and suppose that $\left(\mathcal{A}_{1}, \mathcal{V}_{1}\right),\left(\mathcal{A}_{2}, \mathcal{V}_{2}\right)$ are freely independent. Consider elements $a_{1} \in \mathcal{A}_{1}, a_{2} \in \mathcal{A}_{2}, \xi_{1} \in \mathcal{V}_{1}, \xi_{2} \in \mathcal{V}_{2}$, and denote the $R$-transform of $\left(a_{j}, \xi_{j}\right)$ by $R_{j}$, for $j=1,2$. Then:

(1) The R-transform of $\left(a_{1}, \xi_{1}\right)+\left(a_{2}, \xi_{2}\right)$ is $R_{1}+R_{2}$.

(2) The R-transform of $\left(a_{1}, \xi_{1}\right) \cdot\left(a_{2}, \xi_{2}\right)$ is $R_{1} \otimes^{(B)} R_{2}\left(\right.$ where $\left(a_{1}, \xi_{1}\right) \cdot\left(a_{2}, \xi_{2}\right):=$ $\left(a_{1} a_{2}, a_{1} \xi_{2}+\xi_{1} a_{2}\right)$, as discussed in Section 6.1).

Proof. Let us write explicitly $R_{1}(z)=\sum_{n=1}^{\infty} v_{n} z^{n}$ and $R_{2}(z)=\sum_{n=1}^{\infty} w_{n} z^{n}$, where $v_{n}:=\kappa_{n}^{(B)}\left(\left(a_{1}, \xi_{1}\right), \ldots,\left(a_{1}, \xi_{1}\right)\right), w_{n}:=\kappa_{n}^{(B)}\left(\left(a_{2}, \xi_{2}\right), \ldots,\left(a_{2}, \xi_{2}\right)\right), \quad \forall n \geq 1$.

Part (1) of the proposition amounts to the fact that

$$
\kappa_{n}^{(B)}\left(\left(a_{1}, \xi_{1}\right)+\left(a_{2}, \xi_{2}\right), \ldots,\left(a_{1}, \xi_{1}\right)+\left(a_{2}, \xi_{2}\right)\right)=v_{n}+w_{n}, \forall n \geq 1,
$$

which follows immediately from the multilinearity of the functionals $\kappa_{n}^{(B)}$ and from the condition of vanishing mixed cumulants.

For the proof of part (2), let us denote the moment series and R-transform of $\left(a_{1}, \xi_{1}\right) \cdot\left(a_{2}, \xi_{2}\right)$ by $M$ and $R$, respectively, and let us denote the moment series of $\left(a_{2}, \xi_{2}\right)$ by $M_{2}$. It is sufficient to prove that

$$
M=R_{1} \otimes^{(B)} M_{2} .
$$

Indeed, in view of Proposition 6.5, equation (7.12) amounts to

$$
R \otimes^{(B)} \zeta^{\prime}=R_{1} \star^{(B)} R_{2} \otimes^{(B)} \zeta^{\prime},
$$

and $\zeta^{\prime}$ can be cancelled in (7.13) because it is invertible with respect to $\mathrm{⿴囗}^{(B)}$.

By Theorem 5.3, we know that an equivalent form of (7.12) is

$$
M=R_{1} \otimes_{\mathcal{C}}^{(A)} M_{2} .
$$

On the left-hand side of (7.14), the coefficient of order $n$ is $E\left(\left(\left(a_{1}, \xi_{1}\right) \cdot\left(a_{2}, \xi_{2}\right)\right)^{n}\right)$. By writing $\left(\left(a_{1}, \xi_{1}\right) \cdot\left(a_{2}, \xi_{2}\right)\right)^{n}$ as a product of $2 n$ factors and by using (6.3) of Section 6.2 , we write this coefficient as

$$
\sum_{p \in N C^{(A)}(2 n)} \prod_{\substack{F \text { block } \\ \text { of } p}} \kappa_{\text {card }(F)}^{(B)}\left(\left(a_{1}, \xi_{1}\right),\left(a_{2}, \xi_{2}\right), \ldots,\left(a_{1}, \xi_{1}\right),\left(a_{2}, \xi_{2}\right) \mid F\right) .
$$

Now, due to the condition of vanishing cumulants of type B for $\left(a_{1}, \xi_{1}\right)$ and $\left(a_{2}, \xi_{2}\right)$, we can in fact restrict the summation (7.15) to the partitions $p$ with the property that every block of $p$ is contained either in $\{1,3, \ldots, 2 n-1\}$ or in $\{2,4, \ldots, 2 n\}$. A partition $p \in N C^{(A)}(2 n)$ with the latter property is naturally parametrized by two partitions $p_{1}, p_{2} \in N C^{(A)}(n)$ such that $p_{2} \leq \operatorname{Kr}\left(p_{1}\right)$. (Namely, $p$ is obtained by placing an isomorphic copy of $p_{1}$ on $\{1,3, \ldots, 2 n-1\}$, and an isomorphic copy of $p_{2}$ on $\{2,4, \ldots, 2 n\}$. The condition $p_{2} \leq \operatorname{Kr}\left(p_{1}\right)$ is exactly the one that ensures that $p$ is non-crossing as a partition of $\{1, \ldots, 2 n\}$.) So in the end, the coefficient of order $n$ on the left-hand side of (7.14) gets the form

$$
\sum_{\substack{p_{1}, p_{2} \in N C^{(A)}(n) \\ p_{2} \leq K r\left(p_{1}\right)}}\left(\prod_{\substack{F \text { block } \\ \text { of } p_{1}}} v_{\text {card }(F)}\right) \cdot\left(\prod_{\substack{G \text { block } \\ \text { of } p_{2}}} w_{\text {card }(G)}\right),
$$


or in other words

$$
\sum_{p_{1} \in N C^{(A)}(n)}\left(\prod_{\substack{F \text { block } \\ \text { of } p_{1}}} v_{\operatorname{card}(F)}\right) \cdot\left[\sum_{\substack{p_{2} \in N C^{(A)}(n) \\ p_{2} \leq K r\left(p_{1}\right)}} \prod_{\substack{G \text { block } \\ \text { of } p_{2}}} w_{\text {card }(G)}\right] .
$$

Finally, by using (6.3) again (applied to the couple $\left(a_{2}, \xi_{2}\right)$ ), and the definition of the operation $\Theta_{\mathcal{C}}^{(A)}$, the quantity in (7.16) is easily identified as the coefficient of order $n$ in $R_{1} \otimes_{C}^{(A)} M_{2}$. Thus the coefficients of order $n$ on the two sides of (7.14) are indeed equal to each other (for every $n \geq 1$ ), and this completes the proof.

\section{REFERENCES}

[1] D. Bessis. The dual braid monoid, preprint, January 2002 (arXiv:math.GR/0101158).

[2] P. Biane. Minimal factorizations of a cycle and central multiplicative functions on the infinite symmetric group, Journal of Combinatorial Theory Series A 76 (1996), 197-212. MR 97h:20003

[3] P. Biane. Some properties of crossings and partitions, Discrete Mathematics 175 (1997), 41-53. MR 98h:05020

[4] M. Bozejko and R. Speicher. $\psi$-independent and symmetrized white noises, in Quantum Probability and Related Topics (L. Accardi editor), QP-PQ, VI, World Scientific, Singapore, (1991), 219-236.

[5] T. Brady. A partial order on the symmetric group and new $K(\pi, 1)$ 's for the braid groups, Advances in Mathematics 161 (2001), 20-40. MR 2002k:20066

[6] T. Brady and C. Watt. $K(\pi, 1)$ 's for Artin groups of finite type, preprint, February 2001 (arXiv:math.GR/0102078).

[7] P. Doubilet, G.-C. Rota, and R. Stanley. On the foundations of combinatorial theory (VI): The idea of generating function, Proceedings of the sixth Berkeley symposium on mathematical statistics and probability, Lucien M. Le Cam et al. editors, University of California Press, 1972, 267-318. MR 53:7796

[8] P. de la Harpe. Topics in geometric group theory, University of Chicago Press, 2000. MR 2001i:20081

[9] J. E. Humphreys. Reflection groups and Coxeter groups, Cambridge University Press, 1990. MR 92h:20002

[10] B. Krawczyk and R. Speicher. Combinatorics of free cumulants, Journal of Combinatorial Theory Series A 90 (2000), 267-292. MR 2001f:46101

[11] G. Kreweras. Sur les partitions non croisées d'un cycle, Discrete Mathematics 1 (1972), 333350. MR 46:8852

[12] A. Nica and R. Speicher. A "Fourier transform" for multiplicative functions on non-crossing partitions, Journal of Algebraic Combinatorics 6 (1997), 141-160. MR 98i:46070

[13] A. Nica and R. Speicher. On the multiplication of free $n$-tuples of noncommutative random variables. With an Appendix by D. Voiculescu: Alternative proofs for the type II free Poisson variables and for the compression results. American Journal of Mathematics 118 (1996), 799837. MR 98i:46069

[14] A. Nica and R. Speicher. The combinatorics of free probability, Preliminary version, December 1999. Lecture Notes of Centre Émile Borel, the Henri Poincaré Institute, Paris, France.

[15] A. Nica, D. Shlyakhtenko, and R. Speicher. Operator-valued distributions I. Characterizations of freeness, International Mathematics Research Notices 29 (2002), 1509-1538.

[16] V. Reiner. Non-crossing partitions for classical reflection groups, Discrete Mathematics 177 (1997), 195-222. MR 99f:06005

[17] R. Speicher. Multiplicative functions on the lattice of non-crossing partitions and free convolution, Mathematische Annalen 298 (1994), 611-628. MR 95h:05012

[18] R. Speicher. Combinatorial theory of the free product with amalgamation and operator-valued free probability theory, Memoirs of the Amer. Math. Soc. 132 (1998), no. 627. MR 98i:46071 
[19] D. Voiculescu. Addition of certain noncommuting random variables, Journal of Functional Analysis 66 (1986), 323-346. MR 87j:46122

[20] D. Voiculescu, K. Dykema, and A. Nica. Free random variables, CRM Monograph Series, volume 1, American Mathematical Society, Providence, RI, 1992. MR 94c:46133

Département de Mathématiques et Applications de l'Ecole Normale Supérieure, 45 RUE D'Ulm, 75230 PARIS, France

E-mail address: Philippe.Biane@ens.fr

Department of Mathematics, University of Iowa, Iowa City, Iowa 52242

E-mail address: goodman@math.uiowa.edu

Department of Pure Mathematics, University of Waterloo, Waterloo, Ontario N2L 3G1, CANADA

E-mail address: anica@math.uwaterloo.ca 